\title{
Neoproterozoic High-K Calc-Alkaline Granitoids from Bapa-Batié, North Equatorial Fold Belt, Central Cameroon: Petrogenesis and Geodynamic Significance
}

\author{
Cyrille Chebeu ${ }^{1}$, Christiane Diorlette Ngo Nlend ${ }^{2}$, Jean Paul Nzenti ${ }^{*}, 1$ and Sylvestre Ganno ${ }^{1}$ \\ ${ }^{I}$ Laboratory of Structural Geology and Petrology, Faculty of Science, University of Yaoundé I, P.O. Box 3412 Messa- \\ Yaoundé, Cameroon \\ ${ }^{2}$ Institute of Mineralogy and Geochemistry of Lausanne, University of Lausanne, Switzerland
}

\begin{abstract}
Neoproterozoic granitoids of the Bapa-Batié area crop out within the Central Cameroon Shear Zone (CCSZ). They comprise four rocks: (i) granodiorite, (ii) biotite-granite, (iii) biotite-amphibole-granite, and (iv) granitic dyke. The elongated shape of the plutons and foliation striking parallel to the CCSZ point to syntectonic magma emplacement. The rocks are metaluminous, weakly peraluminous, high-K, and calc-alkaline to shoshonitic ferro-potassic, with mineralogical and geochemical characteristics of I-type granitoids. The rocks are characterized by high $\mathrm{Ba}, \mathrm{Sr}, \mathrm{Zr}, \mathrm{Rb}, \mathrm{Sr}$, low $\mathrm{Ni}$ and $\mathrm{Cr}$ contents, and by variable LREE enrichment, moderate HREE fractionation with strong negative to moderate Eu anomalies (in granodiorite, biotite-granite and biotite-amphibole-granite) and moderate to significant positive Eu anomalies (in granitic enclaves). Trace element distribution patterns show that all these rocks are distinctively depleted in $\mathrm{Nb}, \mathrm{P}, \mathrm{Sr}$ and Ti relative to other trace elements and indicate that these rocks were derived from crustal protoliths. Magmatic zircon grains in these granites yielded LA-ICP-MS U-Pb ages of $600 \pm 3.7 \mathrm{Ma}$ and $619 \pm 29 \mathrm{Ma}$. The plutonic rocks of BapaBatié area resemble other Neoproterozoic high-K calc-alkaline granites of Cameroon and also display strong similarities with high-K calc-alkaline plutons of eastern Nigeria and the Borborema Province in NE Brazil.
\end{abstract}

Keywords: Bapa-Batié neoproterozoic granitoids, central Cameroon shear zone, syntectonic magma emplacement, high-K and calc-alkaline magma, ferro-potassic series, I-type granitoids, LA-ICP-MS U-Pb ages.

\section{INTRODUCTION}

Granitoids display great diversity in their origin, sources and evolution processes and thus can be used as indicators of geodynamic environments and, in some cases as tracers of geodynamic evolution [1, 2]. The Pan-African NorthEquatorial Fold Belt (PANEFB), or Central African Orogen, is a major Neoproterozoic Orogen linked to the TransSaharan Belt of western Africa and to the Brasiliano Orogen of northeastern Brazil (Fig. 1a). In Cameroon, the Neoproterozoic realm [3-10] is subdivided into three domains from south to north (Fig. 1b):

(1) The southern domain comprises Neoproterozoic metasedimentary units, such as the Ntui-Betamba, Yaoundé and Mbalmayo units; the protoliths of these units were deposited in a passive margin environment at the northern edge of the Congo Craton and were metamorphosed under high $\mathrm{P}$ conditions $\left(\mathrm{T}=750-800^{\circ} \mathrm{C}, \mathrm{P}=0.9-1.3 \mathrm{GPa}\right)$ at 616 $\mathrm{Ma}$ [7, 11, 12]. Alkaline magmatism [13] has been also recognized in association with these Neoproterozoic units. The rocks of this southern domain were thrust onto the Archean Congo Craton towards the south [11, 14]. The thrust continues towards the east, forming the Oubanguides Nappe in the Republic of Central Africa.

*Address correspondence to this author at the Laboratory of Structural Geology and Petrology, Faculty of Science, University of Yaoundé I, P.O.Box 3412 Messa-Yaoundé, Cameroon; Tel: + 237948564 73; Emails: jnzenti@uy1.uninet.cm,jnzenti2010@gmail.com
(2) The central domain is positioned between the Sanaga Fault to the south and the Tibati-Banyo Fault to the north. These large NE-striking transcurent faults, as well as the Adamaoua Fault inside the central domain, are regarded as possible prolongations of the major shear zones of NE Brazil in a pre-drift Gondwana reconstruction [15-18]. This central domain consists of Paleoproterozoic high-grade gneisses $\left(850-900^{\circ} \mathrm{C}, 1-1.2 \mathrm{GPa}\right)$ with Archean inheritance in the Palaeoproterozoic gneiss of Meiganga intruded by widespread Neoproterozoic syntectonic plutonic rocks of high-K calc-alkaline Affinities [4-6, 9, 19-24].

(3) The northern domain consists of subordinate $830 \mathrm{Ma}-$ old metavolcanic rocks of tholeiitic and alkaline Affinities associated with metasedimentary rocks known as the Poli Series. Widespread 630-660 Ma calc-alkaline granitoids, now present as orthogneiss, result from a major episode of crustal accretion. A Palaeoproterozoic crustal source in this region is attested by the presence of $2 \mathrm{Ga}$ inherited zircon domains in the granitoids $[6,25]$.

The study area around Bapa-Batié ( 600 km², Fig. 2) belongs to the southwestern part of the central domain. Conventional U-Pb zircon dating has yielded an age of $602 \pm$ 1.4 Ma for this syntectonic Pan-African magmatism in the Batié area [26]. Some geochronological investigations have been carried out on some major magmatic complexes: the Ngondo granitic complex [19], Bandja charnockitic massif [18] and Bangwa granites [25] in the central domain; their emplacement ages vary from $557 \pm 8$ to $686 \pm 17 \mathrm{Ma}(\mathrm{Rb} / \mathrm{Sr}$ 

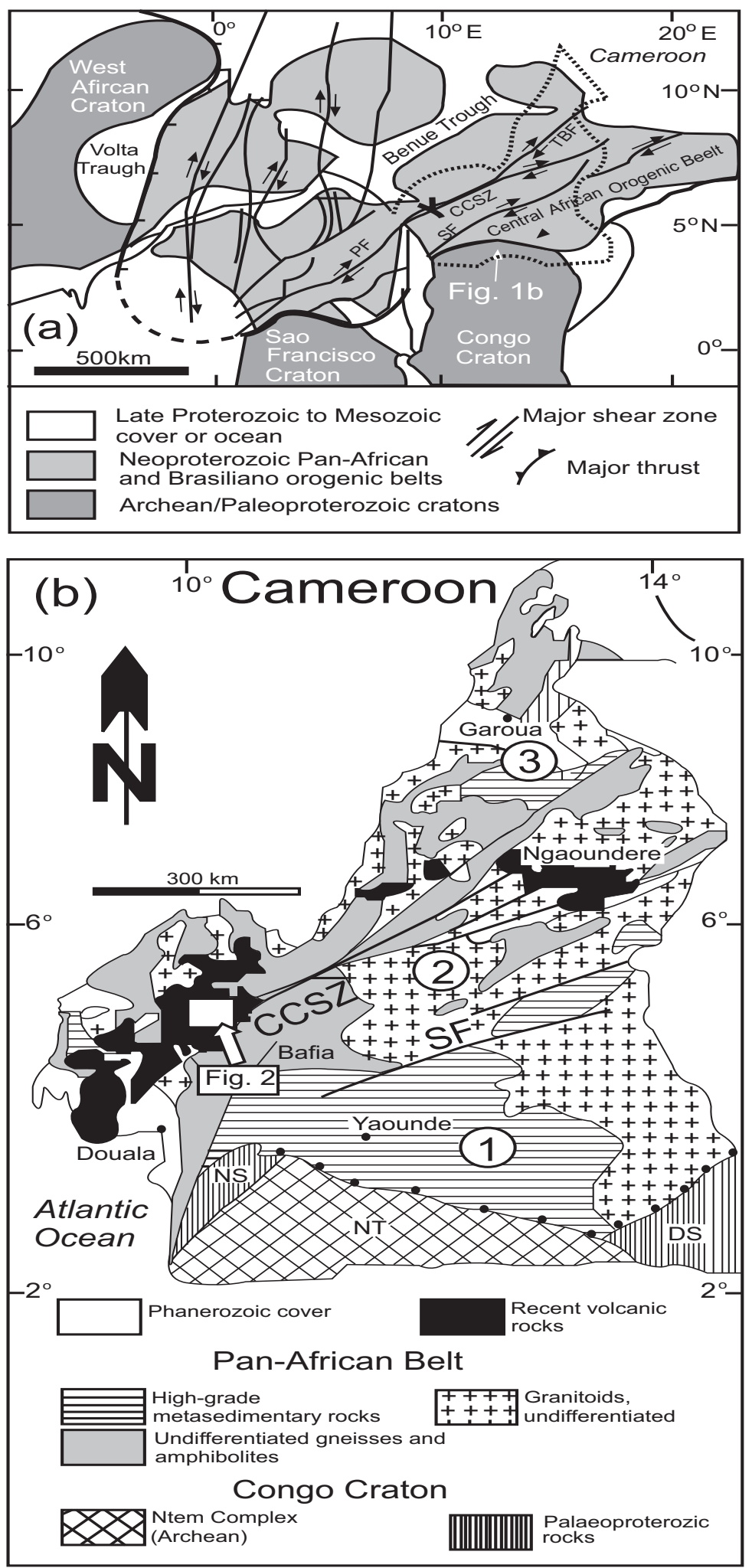

Fig. (1). (a) Geological reconstruction of Africa and NE Brazil (late-Precambrian) Afrter [68]. CCSZ: Cameroon Central Shear Zone; Pa: Patos shear zone; Pe: Pernambuco shear zone; SF: Sanaga fault; TBF: Tibati-Banyo Fault. (b) Geological map of Cameroon with the three main domains of the Pan-African orogenic belt (modified from [4, 69] and adapted from [5, 7, 9]: (1) southern domain corresponding to the Yaoundé series thrusted on the Congo Craton, (2) central domain, (3) northern domain. Granitoids suites are not distinguished due to large scale. CCSZ: Central Cameroon Shear Zone; DS: Dja series; NS: Nyong series; NT: Ntem Complex; SF: Sanaga Fault; TBF: Tibati-Banyo Fault. Location of study is marked by a square. 
whole rock); an U-Pb zircon ages of $640 \pm 15 \mathrm{Ma}$ and $638 \pm 2$ Ma were obtained on the Bandja charnockite and Bangwa granite respectively. All these ages span the early stage of the deformation (orthogneisses) to the late uplift stages (post-tectonic massifs) of the Pan-African fold belt in Cameroon $[9,11,19,26]$.
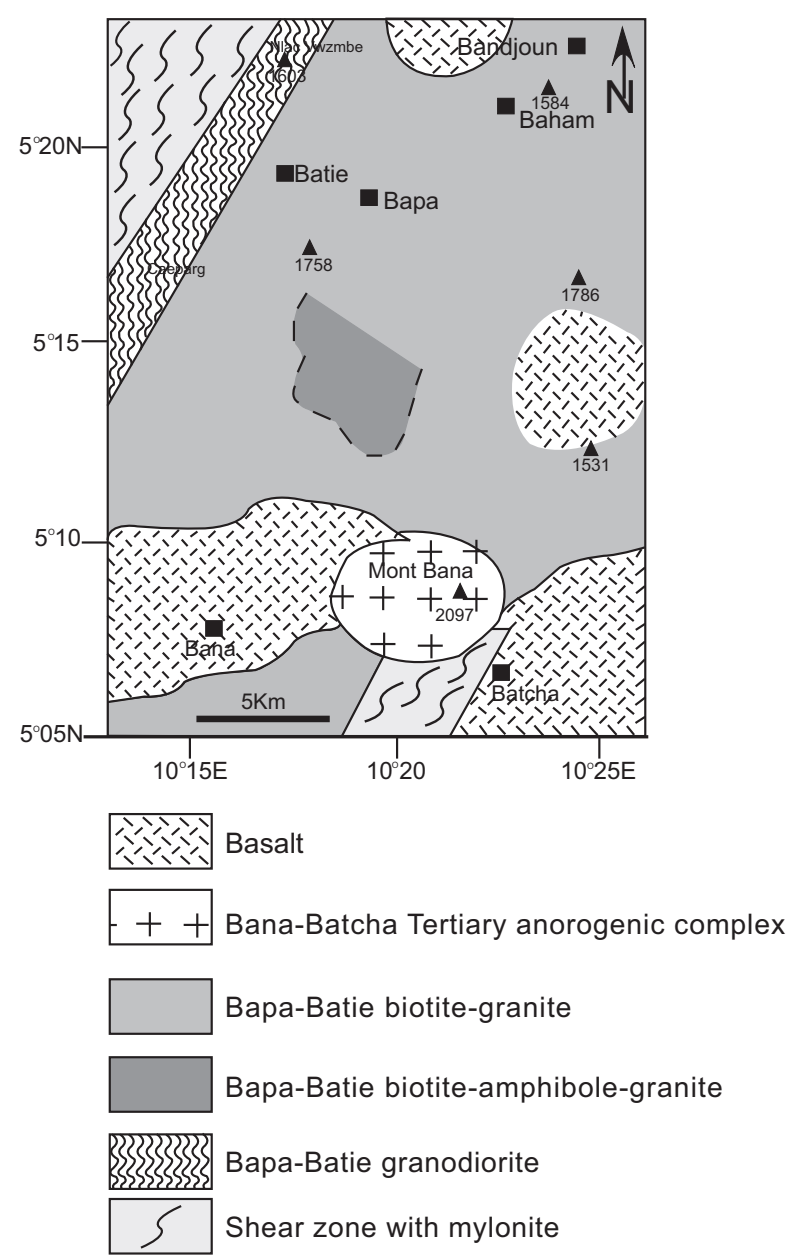

Fig. (2). Simplified geological map of the Bapa-Batié area.

In the present paper, geochemical, mineral chemistry and geochronological data of the plutonic rocks from the BapaBatié area in the southwestern part of the central domain of the PANEFB in Cameroon (Fig. 2) are presented. The aim is to infer the specific geodynamic environment of the granitoids and their geodynamic significance in the PANEFB.

\section{GEOLOGICAL SETTING}

The Batié-Bapa complex $\left(\sim 600 \mathrm{~km}^{2}\right)$ is an NNE- to ENEtrending elliptically shaped massive that is partly deformed and consists of two units (Fig. 2): 1) acidic rocks (biotitegranite, biotite-amphibole-granite) intruded by granitic veins; 2) intermediate rocks (gneissic granodiorite). The plutonic rocks intruded into layered gneiss during Neoproterozoic orogeny [27] at about $600 \mathrm{Ma}$ [25]. The gneissic host rocks are metapelitic and metabasic units of Palaeoproterozoic age $[3,9,23]$ that are grey or dark in color, and display alternating $\mathrm{mm}$ - to $\mathrm{cm}$-scale quartzofelsdpathic and garnet-kyanite-biotite- or garnet-sillimanitebiotite-rich layers. The host rocks were deformed during two main tectonic phases: the first phase $\left(D_{1}\right)$ shows tangential tectonics with NW-SE kinematics direction, and is only recorded in the metamorphic rocks and absent in the granitoids. $D_{1}$ is marked by a flat mylonitic layering $S_{1}$ associated with an $\mathrm{L}_{1}$ stretching lineation and $\mathrm{F}_{1}$ isoclinals intrafolial folds. A mega-fold of regional scale reworked these structures. The second deformation event $\left(\mathrm{D}_{2}\right)$ was a tightening phase with NNE-SSW to ENE-WSW kinematics direction. The $\mathrm{D}_{2}$ phase is recorded in both the metamorphic basement and granitoids. It is characterized by steeply dipping foliation planes roughly parallel to the general strike of the shear zone. The subhorizontal stretching mineral lineation and steep foliation planes are evidence for the dominantly transcurent nature of $\mathrm{D}_{2}$. In the granitoids, the overall conformable nature of the foliation, either acquired during the magmatic stage or at the solid state, point to the syn- $\mathrm{D}_{2}$ emplacement of the studied plutons. Although the strike values of the foliations are very similar, it is worth noting that their dips are less steep in the orthogneiss than in the shear zones proper. Recent work [8] shows that the study area in the central domain experienced transpressive tectonics during the Pan-African orogeny, resulting in a system of "décrochement-nappe" along the $\mathrm{N} 70^{\circ} \mathrm{E}$ shear zone of the Adamaoua with an early sinistral sense of motion $\left(D_{2}\right)$ that was later followed by a dextral sense of shear $\left(D_{3}\right)$. While some workers $[28,29]$ in the eastern part of the Cameroon Central Domain have suggested that $D_{1}$ was a compressional event, $\mathrm{D}_{2}$ also involved crustal compression and a peak metamorphic event. These deformation phases were overprinted by a late Pan-African transpression $\left(\mathrm{D}_{3}\right)$ event. The study area includes the shear zone (Fig. 2): an $\mathrm{N} 30^{\circ} \mathrm{E}$ shear zone passing through Chepang and Nkon Mwambe. It is important to discuss the spatio-temporal relationship of this ancient structure with these granitoids, the nature of the magmas, which produced it, and the significance of this shear zone in the geodynamic context of the Pan-African North Equatorial Fold Belt.

The Bapa-Batié massifs are made up of plutonic rocks, such as granodiorite (orthogneisses), biotite-granite (partly orthogneiss), biotite-amphibole granite and granitic dykes. Outcrops of the former country rocks are scarce, and intrusive contacts with the plutons are generally lacking.

\section{ANALYTICAL METHODS}

Microprobe analyses were carried out at the University of Lausanne with a CAMEBAX SX50. Counting times were 15-30 s per element on peak and 5-30 s on background depending on concentration. The accelerating voltage was 15 $\mathrm{kV}$ for a beam current of 10-20 nA, depending on the sample analyzed. The beam was rastered over a surface of $10 \times 8$ microns for feldspars and $3 \times 2$ microns for hydrated minerals. Natural silicates were used as standards.

Samples for whole-rock major and trace element analyses were crushed and subsequently reduced to a very fine powder by grinding in a tungsten carbide ring mill. Major and trace element concentrations of whole-rock from 34 samples were analyzed by X-ray fluorescence spectrometry 

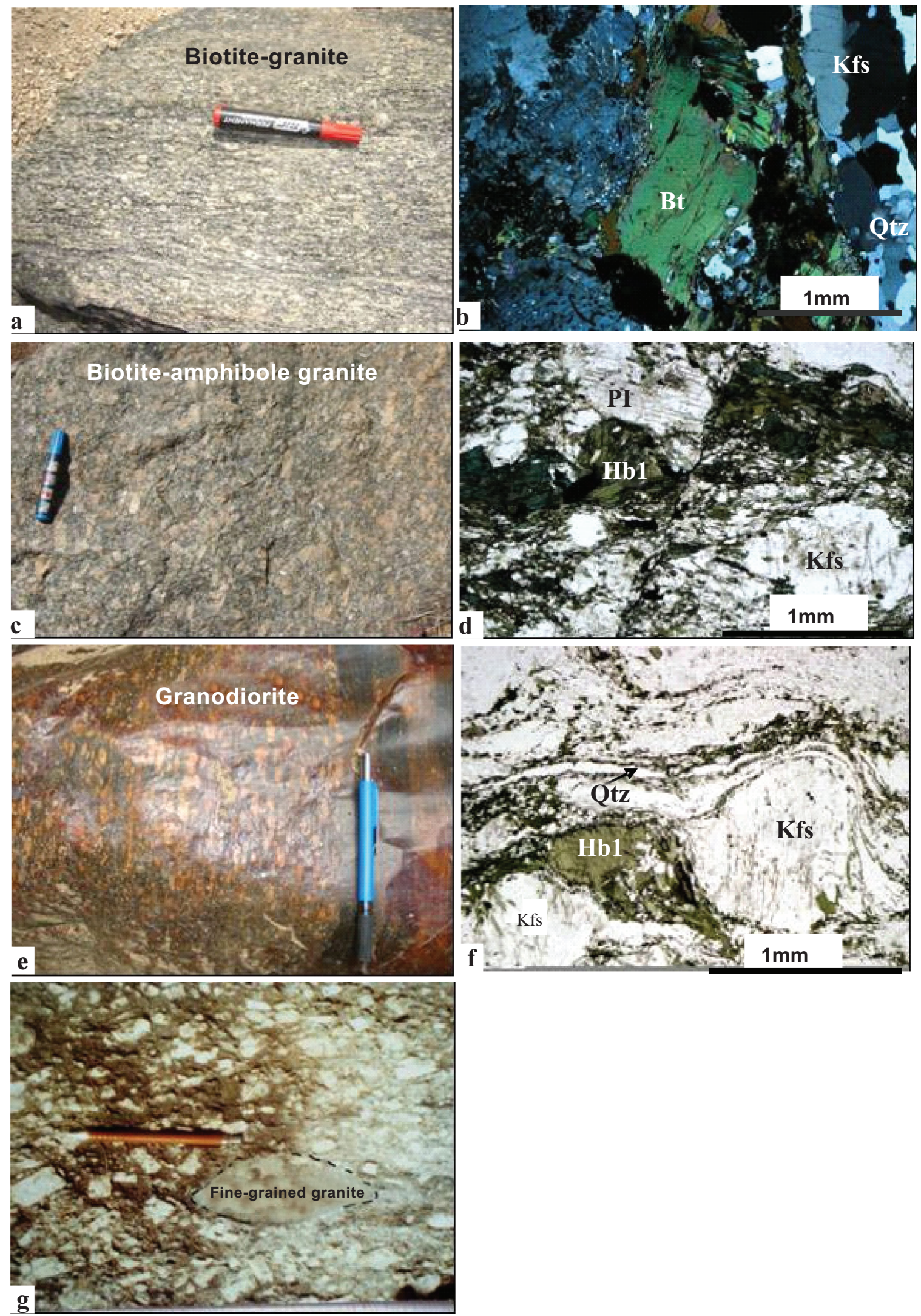

Fig. (3). Field occurrences and microscopic aspects of the granitoids in the Bapa-Batié area. (a) Aspect of Biotite-granite. (b) Detail of texture. (c) Biotite-amphibole- granite with grey pink color and megacrysts of Kfs. (d) Detail of mylonitic foliation with amphibole fish, feldspars porphyroblasts and recrystallisation of quartz. (e) Granodiorite showing strong mylonitic foliation. (f) Photomicrograph of gneissic granodiorite showing asymmetry K-feldspar with a sinistral shearing movement. $(\mathbf{g})$ Fine-grained granite in enclave in biotite-granite. Bt $=$ biotite; Kfs = orthoclase; $\mathrm{pl}=$ plagioclase; $\mathrm{Hbl}=$ hornblende; $\mathrm{Qtz}=$ quartz. 
and LA-ICP-MS, at the University of Lausanne. Major elements ( $\mathrm{Si}, \mathrm{Ti}, \mathrm{Al}, \mathrm{Fe}, \mathrm{Mn}, \mathrm{Mg}, \mathrm{Ca}, \mathrm{Na}, \mathrm{K}, \mathrm{P}, \mathrm{Cr}$ and $\mathrm{Ni}$ ) were measured on fused lithium borate glass disks using a Philips PW2400 X-ray fluorescence spectrometer. Trace elements were measured from pressed powder pellets on the same XRF spectrometer ( $\mathrm{Nb}, \mathrm{Zr}, \mathrm{Y}, \mathrm{Sr}, \mathrm{U}, \mathrm{Rb}, \mathrm{Th}, \mathrm{Pb}, \mathrm{Ga}$, $\mathrm{Ni}, \mathrm{Cr}, \mathrm{V}, \mathrm{Ce}, \mathrm{Ba}$ and $\mathrm{La}$ ), and by LA-ICP-MS on glass disks (Be, Sc, Ti, V, Cr, Ni, Cu, Zn, Y, Zr, Nb, Cs, La, Pr, Nd, Sm, $\mathrm{Eu}, \mathrm{Gd}, \mathrm{Tb}, \mathrm{Dy}, \mathrm{Ho}, \mathrm{Er}, \mathrm{Tm}, \mathrm{Yb}, \mathrm{Lu}, \mathrm{Hf}, \mathrm{Ta}, \mathrm{Pb}$, Th and U). Tests were made to assess the amount of trace element contamination, such as $\mathrm{Ta}$ and $\mathrm{Nb}$, from the tungsten carbide mill. The samples analyses in this study are relatively rich in these elements, therefore contamination is considered negligible. Laser ablation measurements were made with a 193 nm Lambda Physik Excimer laser (Geolas 200M system) coupled to a Perkin-Elmer 6100 DRC ICP-MS. Laser settings were $27 \mathrm{kV}$ with a $10 \mathrm{~Hz}$ repetition rate, yielding a fluorescence of about $12 \mathrm{~J} / \mathrm{cm}^{2}$ on the ablation site. Helium was used as carrier gas $(1.11 / \mathrm{mn})$ and NIST612 glass was used as the external standard, and $\mathrm{Ca}$ and $\mathrm{Al}$ as internal standards (on the basis of electron microprobe measurements on the ablation pit site). BCR2 basaltic glass was regularly used as monitor to check for reproducibility and accuracy of the system. Results were always within $\pm 10 \%$ range of the values reported by Witt-Eickschen et al. (2003) [30], while $\mathrm{Rb}, \mathrm{Cs}, \mathrm{Y}$ and especially $\mathrm{Cr}$ were sometimes of the $\pm 10 \%$ range of the USGS recommended values for BCR2. Analytical uncertainties are currently better than $1 \%$ for major elements and 5-10\% for trace element concentrations higher and lower than 20\%, respectively. Analytical precision for rare earth elements (REE) is estimated at 5\% when concentrations are $>10$ times chondritic and at $10 \%$ when lower.

\section{LA-ICP-MS U-Pb Zircon Age}

Zircon grains were separated using conventional techniques. After crushing and sieving of the powdered samples (250-90 $\mu \mathrm{m}$ fractions), heavy minerals were concentrated by panning and then by conventional heavy liquids and magnetic separation techniques. Non-magnetic crystals at 0 degree of lateral tilt on the isodynamic Frantz separator were examined under a binocular microscope and the most suitable grains were selected for analysis based on their high transparency, lack of mineral inclusions and cracks, euhedral shape and weakest coloration criteria. Zircon grains were heated at $850^{\circ} \mathrm{C}$ in the ambient atmosphere, and then treated with fluoridric acid (HF) and nitric acid $\left(\mathrm{NHO}_{3}\right)$ to remove surface impurities, mounted in epoxy resin and polished. Internal structure and morphology were subsequently observed by Scanning Electron Microscopy (SEM) and cathodoluminescence (CL) imaging using a CAMSCAN MV2300. Data were acquired at the Institute of Geochemistry and Mineralogy, University of Lausanne (Switzerland), using the LA-ICP-MS dating method with a 193mn Ar-F Geollas 200M excimer laser coupled to a Perkin Elmer Elan 6100 DRC. We employed both spot and profiling techniques depending on zircon grain size. Profile laser beam diameter varied between $25 \mu \mathrm{m}$ for grains $<90 \mu \mathrm{m}$ fraction and $35 \mu \mathrm{m}$ for grains $250-90 \mu \mathrm{m}$. Typical internal precision varied between 2 and $5 \%$ for the calculation of ${ }^{207} \mathrm{~Pb} /{ }^{235} \mathrm{U}$ and ${ }^{206} \mathrm{~Pb} /{ }^{238} \mathrm{U}$ ages, respectively, and was independent of the applied measurement mode. External well-characterized zircon reference standards (91500 and Plesovice) were monitored to measure routinely our grains during analytical session. The age obtained for the 91500 zircon standard is $1069.4 \pm 5.9 \mathrm{MA}(2 \sigma, 23$ analyses $)$ that for the Plesovice standard is $337.8 \pm 1.9 \mathrm{MA}(2 \sigma, 34$ analyses).

\section{RESULTS}

\section{Petrography and Mineral Composition}

\section{Biotite-Granite}

Biotite-granite (Fig. 3a, b) is the most abundant rock type and consist of quartz (30-40 vol-\%), plagioclase (10-15 vol$\%)$, K-feldspar (20-30 vol-\%), biotite (10-15 vol- \%), accessory minerals (zircon, titanite, apatite) occur in very small proportions. Zircon is commonly present as inclusions in biotite and K-feldspar. Grain sizes are generally about a few millimeters, reaching as much as $2 \mathrm{~cm}$; most samples are porphyritic with feldspar crystals up to $4 \mathrm{~cm}$ in length. Quartz occurs as polycrystalline ribbon with irregular grain boundaries or subgrain boundaries. Plagioclase $\left(\mathrm{An}_{17-27}\right.$, Table 1) occurs as partly elongated, subhedral or rounded crystals. In some samples, plagioclase has inclusions of small euhedral biotite and zircon crystals. K-feldspar $\left(\mathrm{Or}_{90}\right.$ 95, Table 2) exhibit perthitic intergrowth; crystals are subhedral or rounded and oriented. K-feldspar has inclusions of small euhedral biotite, apatite and zircon crystals. Biotite $\left(\mathrm{X}_{\mathrm{Mg}}=0.39-0.54\right.$, Table 3$)$ occurs as flakes of various dimensions that may be clustered, most of which have corroded margins. Some crystals are kinked. Biotite has inclusions of small euhedral zircon and titanite crystals.

\section{Biotite-Amphibole Granite}

Biotite-amphibole-granite (Fig. 3c, d) occurs as coarsegrained, grey-pink rock evenly grained with the following modal composition: quartz (35-45 vol-\%), K-feldspar (20-25 vol- $\%)$, plagioclase (5-10 vol- $\%)$, biotite (5-10 vol- $\%)$, amphibole (10-20 vol-\%) and titanite. Quartz is present as a large crystals or aggregates of quartz grains with undulose extinction. K-feldspar is orthoclase $\left(\mathrm{Or}_{91-92}\right.$, Table 2), occurs as phenocrysts with as much as $4 \mathrm{~cm}$ length and exhibits, in places, perthitic exsolution. Plagioclase $\left(\mathrm{An}_{19-21}\right.$, Table 1) forms sub- to anhedral grains (1.5 to $4 \mathrm{~mm}$ in length). Some grains show a bulbous symplectite of vermicular quartz (myrmekite). Plagioclase contains inclusions of apatite and zircon. Green hornblende (magnesiokatophorite, Table 4) occurs as anhedral grains with development of minute grains of quartz and biotite at the grain boundaries. Amphibole contains numerous apatite and titanite inclusions. Biotite $\left(\mathrm{X}_{\mathrm{Mg}}=0.54-0.57\right.$, Table 3$)$ is present as lamellae replacing amphibole and contains small euhedral zircon inclusion. Titanite forms lozenge aggregates associated with amphibole and biotite.

\section{Granodiorite}

Gneissic granodiorite (Fig. 3e, f) occupy the centre of the Chepang shear zone (elongate domain) in the northwestern area of the massif. In the field, granodiorite is usually a medium-grained, dark rock. It is characterized by pressure shadows on sides of K-feldspar megacrystals, strong planar- 
Table 1. Representative Analyses of Plagioclase from Bapa-Batié Plutonic Rocks (wt\%). Number of Ions on the Basis of 8 Oxygens

\begin{tabular}{|c|c|c|c|c|c|c|c|c|c|c|c|c|c|c|c|c|c|c|c|c|c|c|c|c|}
\hline \multirow{2}{*}{$\overline{\mathrm{N}^{\circ}}$} & \multicolumn{7}{|c|}{ Plagioclase } & \multicolumn{9}{|c|}{ Granodiorite } & \multicolumn{4}{|c|}{ Biotite- Granite } & \multicolumn{4}{|c|}{ Biotite-Amphibole Granite } \\
\hline & 21 & 22 & 23 & 24 & 25 & 26 & 27 & 28 & 29 & 30 & 31 & 32 & 33 & & & 36 & 37 & 38 & 39 & 40 & 41 & 42 & 43 & 44 \\
\hline $\mathrm{SiO}_{2}$ & 59.79 & 59.58 & 60.01 & 59.97 & 60.13 & 60.01 & 59.87 & 59.81 & 61.03 & 60.07 & 60.09 & 60.16 & 60.58 & 61.13 & 60.47 & 60.26 & 62.95 & 60.99 & 63.72 & 63.54 & 62.01 & 61.81 & 62.15 & 62.51 \\
\hline $\mathrm{Al}_{2} \mathrm{O}_{3}$ & 24.05 & 23.91 & 23.99 & 23.11 & 23.38 & 24.03 & 24.89 & 24.13 & 24.89 & 24.97 & 23.87 & 25.01 & 24.85 & 24.71 & 23.63 & 23.77 & 23.03 & 24.54 & 22.75 & 23.01 & 23.56 & 23.46 & 24.05 & 23.31 \\
\hline $\mathrm{CaO}$ & 11.96 & 11.22 & 11.03 & 12.05 & 12.03 & 10.01 & 6.89 & 10.31 & 4.91 & 6.01 & 6.66 & 6.52 & 5.96 & 5.88 & 6.31 & 6.25 & 4.29 & 5.73 & 3.63 & 3.92 & 4.69 & 4.87 & 4.44 & 4.21 \\
\hline $\mathrm{Na}_{2} \mathrm{O}$ & 4.91 & 5.66 & 5.76 & 4.96 & 4.81 & 6.01 & 8.09 & 5.71 & 8.83 & 7.98 & 9.58 & 8.38 & 8.61 & 8.01 & 9.07 & 9.65 & 9.51 & 8.63 & 9.74 & 9.46 & 9.34 & 9.76 & 9.45 & 9.65 \\
\hline $\mathrm{K}_{2} \mathrm{O}$ & 0.16 & 0.18 & 0.11 & 0.13 & 0.21 & 0.23 & 0.55 & 0.21 & 0.35 & 0.53 & 0.11 & 0.13 & 0.21 & 0.25 & 0.17 & 0.15 & 0.09 & 0.19 & 0.13 & 0.11 & 0.21 & 0.24 & 0.19 & 0.31 \\
\hline Total & 100.87 & 100.55 & 100.9 & 100.22 & 100.56 & 100.29 & 100.29 & 100.17 & 100.01 & 99.56 & 100.31 & 100.2 & 100.21 & 99.98 & 99.65 & 100.08 & 99.87 & 100.08 & 99.97 & 100.04 & 99.81 & 100.14 & 100.28 & 99.99 \\
\hline $\mathrm{Si}$ & 2.6607 & 2.662 & 2.6686 & 2.6863 & 2.6832 & 2.679 & 2.671 & 2.6732 & 2.7108 & 2.6877 & 2.6875 & 2.6778 & 2.6931 & 2.7144 & 2.712 & 2.6979 & 2.7902 & 2.7108 & 2.8152 & 2.8053 & 2.7578 & 2.7442 & 2.5627 & 2.7712 \\
\hline $\mathrm{Al}$ & 1.2613 & 1.2591 & 1.2573 & 1.22 & 1.2296 & 1.2643 & 1.3087 & 1.2711 & 1.303 & 1.3168 & 1.2582 & 1.312 & 1.302 & 1.2931 & 1.249 & 1.2542 & 1.2031 & 1.2855 & 1.1846 & 1.1973 & 1.2349 & 1.2276 & 1.1688 & 1.2179 \\
\hline $\mathrm{Ca}$ & 0.5703 & 0.5371 & 0.5255 & 0.5783 & 0.5752 & 0.4788 & 0.3293 & 0.4937 & 0.2337 & 0.2881 & 0.3191 & 0.311 & 0.2839 & 0.2797 & 0.3032 & 0.2998 & 0.2037 & 0.2729 & 0.1718 & 0.1854 & 0.2235 & 0.2317 & 0.1962 & 0.2 \\
\hline $\mathrm{K}$ & 0.0091 & 0.0103 & 0.0062 & 0.0074 & 0.012 & 0.0131 & 0.0313 & 0.012 & 0.0198 & 0.0303 & 0.0063 & 0.0074 & 0.0119 & 0.0142 & 0.0097 & 0.0086 & 0.0051 & 0.0108 & 0.0073 & 0.0062 & 0.0119 & 0.0136 & 0.01 & 0.0175 \\
\hline $\mathrm{Na}$ & 0.4236 & 0.4903 & 0.4966 & 0.4308 & 0.4162 & 0.5202 & 0.6998 & 0.4948 & 0.7604 & 0.6923 & 0.8307 & 0.7232 & 0.7421 & 0.6896 & 0.7887 & 0.8377 & 0.8173 & 0.7437 & 0.8343 & 0.8098 & 0.8054 & 0.8401 & 0.7555 & 0.8294 \\
\hline $\mathrm{X}$ an & 0.57 & 0.52 & 0.51 & 0.57 & 0.57 & 0.47 & 0.31 & 0.49 & 0.23 & 0.29 & 0.28 & 0.30 & 0.27 & 0.28 & 0.28 & 0.26 & 0.20 & 0.27 & 0.17 & 0.19 & 0.21 & 0.21 & 0.20 & 0.19 \\
\hline $\mathrm{X}$ ab & 0.42 & 0.47 & 0.48 & 0.42 & 0.41 & 0.51 & 0.66 & 0.49 & 0.75 & 0.68 & 0.72 & 0.69 & 0.72 & 0.70 & 0.72 & 0.73 & 0.80 & 0.72 & 0.82 & 0.81 & 0.77 & 0.77 & 0.79 & 0.79 \\
\hline $\mathrm{X}$ or & 0.01 & 0.01 & 0.01 & 0.01 & 0.01 & 0.01 & 0.03 & 0.01 & 0.02 & 0.03 & 0.01 & 0.01 & 0.01 & 0.01 & 0.01 & 0.01 & 0.00 & 0.01 & 0.01 & 0.01 & 0.01 & 0.01 & 0.01 & 0.02 \\
\hline & & Labr & rador & & & Ande & esine & & Oligo & clase & & & & & & & & Oligo & clase & & & Oligo & oclase & \\
\hline
\end{tabular}

Table 2. Representative Analyses of K-Feldspar from Bapa-Batié Plutonic Rocks (wt\%). Number of Ions on the Basis of 8 Oxygens

\begin{tabular}{|c|c|c|c|c|c|c|c|c|c|c|c|c|c|c|c|c|c|c|c|}
\hline \multicolumn{8}{|c|}{ Granodiorite } & \multicolumn{7}{|c|}{ Biotite-Granite } & \multicolumn{5}{|c|}{ Biotite-Amphibole Granite } \\
\hline $\mathrm{N}^{\circ}$ & 1 & 2 & 3 & 4 & 5 & 6 & 7 & 8 & 10 & 11 & 12 & 13 & 14 & 15 & 16 & 17 & 18 & 19 & 20 \\
\hline $\mathrm{SiO}_{2}$ & 65.04 & 65.13 & 65.23 & 64.98 & 64.91 & 65.11 & 64.98 & 63.89 & 64.74 & 64.91 & 64.04 & 65.03 & 64.65 & 64.85 & 64.91 & 65.03 & 65.01 & 64.04 & 64.45 \\
\hline $\mathrm{Al}_{2} \mathrm{O}_{3}$ & 19.02 & 18.86 & 18.87 & 18.95 & 19.03 & 19.03 & 18.86 & 18.72 & 18.65 & 18.48 & 18.74 & 18.65 & 18.96 & 18.86 & 18.55 & 18.43 & 18.64 & 18.87 & 18.94 \\
\hline $\mathrm{CaO}$ & 0.02 & 0.03 & 0.01 & 0 & 0.01 & 0.03 & 0.01 & 0 & 0 & 0 & 0 & 0 & 0 & 0 & 0 & 0 & 0 & 0 & 0 \\
\hline $\mathrm{Na}_{2} \mathrm{O}$ & 0.71 & 1.01 & 0 & 0.81 & 0.75 & 0.79 & 1.01 & 0.56 & 1.12 & 0.56 & 0.58 & 0.56 & 0.51 & 0.76 & 0.86 & 0.91 & 0.91 & 1.02 & 0.98 \\
\hline $\mathrm{K}_{2} \mathrm{O}$ & 15.29 & 15.31 & 15.93 & 15.21 & 15.46 & 15.22 & 15.36 & 16.42 & 15.24 & 15.91 & 16.19 & 15.98 & 15.76 & 15.58 & 15.84 & 15.65 & 15.63 & 16.25 & 15.69 \\
\hline Total & 100.08 & 100.34 & 100.04 & 99.95 & 100.16 & 100.2 & 100.22 & 99.59 & 99.75 & 99.86 & 99.55 & 100.22 & 99.88 & 100.05 & 100.16 & 100.02 & 100.19 & 100.18 & 100.06 \\
\hline $\mathrm{Si}$ & 2.9875 & 2.9066 & 2.7925 & 2.9283 & 2.8199 & 2.9135 & 2.9014 & 2.9735 & 2.9893 & 2.9985 & 2.9769 & 2.9939 & 2.9833 & 2.9865 & 2.9921 & 2.9984 & 2.9920 & 2.9649 & 2.9745 \\
\hline $\mathrm{Al}$ & 1.0296 & 1.1018 & 1.2338 & 1.0883 & 1.1965 & 1.1039 & 1.1067 & 1.0268 & 1.0149 & 1.0061 & 1.0267 & 1.0119 & 1.0311 & 1.0236 & 1.0078 & 1.0015 & 1.0111 & 1.0296 & 1.0302 \\
\hline $\mathrm{Ca}$ & 0.0010 & 0.0016 & 0.0006 & 0.0000 & 0.0006 & 0.0016 & 0.0005 & 0.0000 & 0.0000 & 0.0000 & 0.0000 & 0.0000 & 0.0000 & 0.0000 & 0.0000 & 0.0000 & 0.0000 & 0.0000 & 0.0000 \\
\hline $\mathrm{K}$ & 0.8960 & 0.9681 & 1.1274 & 0.9455 & 1.0522 & 0.9557 & 0.9756 & 0.9749 & 0.8977 & 0.9376 & 0.9601 & 0.9386 & 0.9278 & 0.9153 & 0.9315 & 0.9205 & 0.9177 & 0.9598 & 0.9238 \\
\hline $\mathrm{Na}$ & 0.0632 & 0.0971 & 0.0000 & 0.0765 & 0.0776 & 0.0754 & 0.0975 & 0.0505 & 0.1003 & 0.0502 & 0.0523 & 0.0500 & 0.0456 & 0.0679 & 0.0769 & 0.0814 & 0.0812 & 0.0916 & 0.0877 \\
\hline $\mathrm{X}$ or & 0.93 & 0.91 & 0.99 & 0.93 & 0.93 & 0.93 & 0.91 & 0.95 & 0.90 & 0.95 & 0.95 & 0.95 & 0.95 & 0.93 & 0.92 & 0.92 & 0.92 & 0.91 & 0.91 \\
\hline $\mathrm{X}$ an & 0.00 & 0.00 & 0.00 & 0.00 & 0.00 & 0.00 & 0.00 & 0.00 & 0.00 & 0.00 & 0.00 & 0.00 & 0.00 & 0.00 & 0 & 0 & 0 & 0 & 0 \\
\hline $\mathrm{X} a b$ & 0.07 & 0.09 & 0.01 & 0.07 & 0.07 & 0.07 & 0.09 & 0.05 & 0.10 & 0.05 & 0.05 & 0.05 & 0.05 & 0.07 & 0.08 & 0.08 & 0.08 & 0.09 & 0.09 \\
\hline
\end{tabular}

preferred orientation of biotite and amphibole, and quartz grains flattened and/or elongate, and a common development of C-S structures. It is composed of quartz (15-25 vol-\%), Kfeldspar (10-15 vol-\%), plagioclase (50-60 vol-\%), biotite (15-20 vol-\%), amphibole (5-10 vol-\%); Accessory minerals include titanite and oxides ( 2 vol- $\%)$. Quartz forms oriented single or platy crystals, polycrystalline ribbons or small rounded ones $\left(0.1-0.3 \mathrm{~mm}\right.$ in diameter). K-feldspar $\left(\mathrm{Or}_{91-99}\right.$, Table 2) occurs as elongate crystals $(6 \times 3 \mathrm{~mm}$ to $0.4 \mathrm{x}$ $0.2 \mathrm{~mm}$ ) or almond-shape with small flakes of biotite in inclusion. Bulbous symplectite of vermicular quartz (myrmekite) in K-feldspar is common. Tabular plagioclase $\left(\mathrm{An}_{23-49}\right.$, Table 1) grains vary in size, reaching as much as $2 \mathrm{~mm}$, and have inclusions of small grains of quartz and biotite. Biotite $\left(\mathrm{X}_{\mathrm{Mg}}=0.47-0.54\right.$, Table 3 ) forms platy flakes ( $2 \times 0.8 \mathrm{~mm}$ to $0.8 \times 0.3 \mathrm{~mm})$ and has inclusions of euhedral titanite. Green hornblende grains (katophorite and magnesiokatophorite, Table 4) are up to $1.5 \mathrm{~mm}$ in the length, show retrograde alteration to biotite and oxides, and 
Table 3. Representative Analyses of Biotite from Bapa-Batié Plutonic Rocks (wt\%). Number of Ions on the Basis of 22 Oxygens

\begin{tabular}{|c|c|c|c|c|c|c|c|c|c|c|c|c|c|c|c|c|c|c|c|c|}
\hline & \multicolumn{13}{|c|}{ Biotite-Granite } & \multicolumn{7}{|c|}{ Biotite-Amphibole Granite } \\
\hline $\mathrm{N}^{\circ}$ & 20 & 21 & 22 & 23 & 24 & 25 & 26 & 27 & 28 & 29 & 30 & 31 & 32 & 33 & 34 & 35 & 36 & 37 & 38 & 39 \\
\hline $\mathrm{SiO}_{2}$ & 36.45 & 36.07 & 35.99 & 35.69 & 35.74 & 36.27 & 35.46 & 36.77 & 36.11 & 36.39 & 36.67 & 36.17 & 36.41 & 36.01 & 36.05 & 36.05 & 36.51 & 36.63 & 35.98 & 36.01 \\
\hline $\mathrm{TiO}_{2}$ & 1.75 & 2.44 & 2.46 & 2.93 & 2.38 & 2.66 & 2.81 & 2.54 & 2.66 & 2.91 & 2.87 & 2.71 & 2.83 & 1.38 & 1.51 & 1.56 & 1.63 & 1.65 & 2.21 & 2.23 \\
\hline $\mathrm{Al}_{2} \mathrm{O}_{3}$ & 16.31 & 15.96 & 16.63 & 16.44 & 16.36 & 15.93 & 15.98 & 16.11 & 16.03 & 16.13 & 16.21 & 15.98 & 16.01 & 15.11 & 15.05 & 15.21 & 15.11 & 15.45 & 15.33 & 15.44 \\
\hline $\mathrm{FeO}$ & 21.01 & 22.94 & 22.99 & 22.4 & 23.56 & 23.02 & 23.07 & 23.01 & 23.58 & 22.85 & 22.51 & 24.27 & 24.09 & 19.13 & 19.22 & 19.21 & 19.43 & 19.35 & 19.85 & 19.82 \\
\hline $\mathrm{MgO}$ & 11.13 & 9.68 & 9.52 & 9.55 & 9.51 & 8.44 & 8.96 & 9.01 & 9.34 & 9.51 & 9.66 & 8.69 & 9.01 & 14.26 & 14.19 & 13.67 & 14.11 & 13.99 & 13.98 & 14.35 \\
\hline $\mathrm{MnO}$ & 0.21 & 0.31 & 0.34 & 0.28 & 0.21 & 0.29 & 0.26 & 0.34 & 0.31 & 0.34 & 0.28 & 0.29 & 0.31 & 0.35 & 0.31 & 0.35 & 0.28 & 0.25 & 0.31 & 0.36 \\
\hline $\mathrm{K}_{2} \mathrm{O}$ & 9.34 & 9.32 & 9.35 & 9.58 & 9.59 & 9.43 & 9.47 & 9.78 & 9.61 & 9.88 & 9.76 & 9.87 & 9.41 & 9.65 & 9.65 & 9.26 & 9.34 & 9.53 & 9.51 & 9.44 \\
\hline Total & 96.2 & 96.72 & 97.28 & 96.87 & 97.35 & 96.04 & 96.01 & 97.56 & 97.64 & 98.01 & 97.96 & 97.98 & 98.07 & 95.89 & 95.98 & 95.31 & 96.41 & 96.85 & 97.17 & 97.65 \\
\hline $\mathrm{Mg}$ & 0.4305 & 0.4541 & 0.3713 & 0.4541 & 0.3704 & 0.4541 & 0.3481 & 0.4541 & 0.3658 & 0.4541 & 0.3783 & 0.4541 & 0.3518 & 0.5374 & 0.4541 & 0.5194 & 0.4541 & 0.5229 & 0.4541 & 0.5294 \\
\hline $\mathrm{Fe}$ & 0.4558 & 0.3897 & 0.503 & 0.3897 & 0.5148 & 0.3897 & 0.5464 & 0.3897 & 0.518 & 0.3897 & 0.4945 & 0.3897 & 0.5277 & 0.4044 & 0.3897 & 0.4094 & 0.3897 & 0.4057 & 0.3897 & 0.4102 \\
\hline $\mathrm{Ti}$ & 0.0341 & 0.0287 & 0.0484 & 0.0287 & 0.0468 & 0.0287 & 0.0551 & 0.0287 & 0.0526 & 0.0287 & 0.0567 & 0.0287 & 0.0558 & 0.0262 & 0.0287 & 0.0299 & 0.0287 & 0.0311 & 0.0287 & 0.0415 \\
\hline AlVI & 0.0796 & 0.1276 & 0.0773 & 0.1276 & 0.0679 & 0.1276 & 0.0505 & 0.1276 & 0.0636 & 0.1276 & 0.0705 & 0.1276 & 0.0647 & 0.032 & 0.1276 & 0.0413 & 0.1276 & 0.0403 & 0.1276 & 0.0189 \\
\hline $\mathrm{K}$ & 1 & 0.9782 & 1 & 0.9782 & 1 & 0.9782 & 1 & 0.9782 & 1 & 0.9782 & 1 & 0.9782 & 1 & 1 & 0.9782 & 1 & 0.9782 & 1 & 0.9782 & 1 \\
\hline$X_{\mathrm{Mg}}$ & 0.49 & 0.54 & 0.42 & 0.54 & 0.42 & 0.54 & 0.39 & 0.54 & 0.41 & 0.54 & 0.43 & 0.54 & 0.40 & 0.57 & 0.54 & 0.56 & 0.54 & 0.56 & 0.54 & 0.56 \\
\hline
\end{tabular}

\begin{tabular}{|c|c|c|c|c|c|c|c|c|c|c|c|c|c|c|c|c|c|c|c|}
\hline \multicolumn{20}{|c|}{ Granodiorite } \\
\hline $\mathrm{N}^{\circ}$ & 1 & 2 & 3 & 4 & 5 & 6 & 7 & 8 & 9 & 10 & 11 & 12 & 13 & 14 & 15 & 16 & 17 & 18 & 19 \\
\hline $\mathrm{SiO}_{2}$ & 36.71 & 35.97 & 36.81 & 36.51 & 36.61 & 36.17 & 36.36 & 36.54 & 36.35 & 36.41 & 36.56 & 36.51 & 36.71 & 36.78 & 36.65 & 36.08 & 36.53 & 36.21 & 36.41 \\
\hline $\mathrm{TiO}_{2}$ & 1.89 & 1.81 & 1.75 & 1.68 & 1.63 & 1.91 & 1.96 & 1.76 & 1.78 & 1.75 & 1.73 & 1.79 & 1.91 & 1.96 & 1.97 & 1.99 & 1.81 & 1.75 & 1.81 \\
\hline $\mathrm{Al}_{2} \mathrm{O}_{3}$ & 14.75 & 14.84 & 14.56 & 14.61 & 14.45 & 14.71 & 14.52 & 14.38 & 14.64 & 14.51 & 14.61 & 14.56 & 14.79 & 14.77 & 14.31 & 14.35 & 14.21 & 14.62 & 14.41 \\
\hline $\mathrm{FeO}$ & 21.51 & 21.38 & 21.71 & 21.52 & 21.12 & 20.98 & 21.19 & 20.99 & 21.37 & 20.97 & 20.91 & 21.38 & 21.01 & 21.32 & 20.56 & 20.44 & 20.83 & 20.11 & 20.91 \\
\hline $\mathrm{MgO}$ & 10.55 & 10.85 & 10.96 & 10.96 & 10.86 & 10.84 & 10.85 & 11.15 & 11.35 & 11.22 & 11.12 & 11.01 & 10.98 & 11.01 & 11.36 & 11.24 & 11.55 & 11.81 & 11.15 \\
\hline $\mathrm{MnO}$ & 0.21 & 0.23 & 0.21 & 0.28 & 0.31 & 0.33 & 0.35 & 0.25 & 0.34 & 0.37 & 0.31 & 0.33 & 0.27 & 0.23 & 0.31 & 0.35 & 0.33 & 0.21 & 0.25 \\
\hline $\mathrm{K}_{2} \mathrm{O}$ & 9.38 & 9.77 & 9.78 & 9.87 & 9.84 & 9.85 & 9.75 & 9.86 & 9.74 & 9.88 & 9.77 & 9.61 & 9.57 & 9.71 & 10.44 & 10.51 & 10.87 & 10.56 & 9.55 \\
\hline Total & 95.00 & 94.85 & 95.78 & 95.43 & 94.82 & 94.79 & 94.98 & 94.93 & 95.57 & 95.11 & 95.01 & 95.19 & 95.24 & 95.78 & 95.6 & 94.96 & 96.13 & 95.27 & 94.49 \\
\hline $\mathrm{Mg}$ & 0.4192 & 0.4541 & 0.4321 & 0.4541 & 0.4349 & 0.4541 & 0.4337 & 0.4541 & 0.4465 & 0.4541 & 0.4424 & 0.4541 & 0.4351 & 0.4541 & 0.4544 & 0.4541 & 0.4608 & 0.4541 & 0.444 \\
\hline $\mathrm{Fe}$ & 0.4795 & 0.3897 & 0.4801 & 0.3897 & 0.4745 & 0.3897 & 0.4751 & 0.3897 & 0.4716 & 0.3897 & 0.4667 & 0.3897 & 0.467 & 0.3897 & 0.4614 & 0.3897 & 0.4662 & 0.3897 & 0.4671 \\
\hline $\mathrm{Ti}$ & 0.0379 & 0.0287 & 0.0348 & 0.0287 & 0.0329 & 0.0287 & 0.0395 & 0.0287 & 0.0353 & 0.0287 & 0.0347 & 0.0287 & 0.0382 & 0.0287 & 0.0398 & 0.0287 & 0.0364 & 0.0287 & 0.0364 \\
\hline AlVI & 0.0634 & 0.1276 & 0.053 & 0.1276 & 0.0577 & 0.1276 & 0.0517 & 0.1276 & 0.0466 & 0.1276 & 0.0561 & 0.1276 & 0.0597 & 0.1276 & 0.0444 & 0.1276 & 0.0366 & 0.1276 & 0.0525 \\
\hline $\mathrm{K}$ & 1 & 0.9782 & 1 & 0.9782 & 1 & 0.9782 & 1 & 0.9782 & 1 & 0.9782 & 1 & 0.9782 & 1 & 0.9782 & 1 & 0.9782 & 1 & 0.9782 & 1 \\
\hline $\mathrm{X}_{\mathrm{Mg}}$ & 0.47 & 0.54 & 0.47 & 0.54 & 0.48 & 0.54 & 0.48 & 0.54 & 0.49 & 0.54 & 0.49 & 0.54 & 0.48 & 0.54 & 0.50 & 0.54 & 0.50 & 0.54 & 0.49 \\
\hline
\end{tabular}

contain inclusion of titanite, Fe-oxides, apatite, biotite and zircon.

\section{Fine-Grained Granite}

Fine-grained granite occurs as enclaves in the biotite and biotite-amphibole granite (Fig. 3g). This granite is finegrained, dark-grey in color, and is made up of quartz (25-35 vol-\%), K-feldspar (25-35 vol-\%), plagioclase (8-15 vol-\%), and biotite (5-12 vol- $\%)$.

\section{Geochemistry}

Representative major and trace element data for samples of granitoids of the magmatic complex of Bapa-Batié are listed in Table 5.

\section{Major Elements}

In the QAPF diagram (Fig. 4, [70]), the Bapa-Batié plutonic rocks fall in the granite and granodiorite fields. $\mathrm{SiO}_{2}$ contents range from 68.87-72.89 wt\% in biotite-granite and 70.23-74.74 wt\% in biotite-amphibole-granite, 62.2-66.88 $\mathrm{wt} \%$ in granodiorite and 69.07 to $72.16 \mathrm{wt} \%$ for fine-grained granite (syenogranite). Major element variations for BapaBatié plutonic rocks are shown on selected oxide diagrams (Fig. 5). The biotite-granite samples show some slight chemical differences with other samples, in particular their much lower content of $\mathrm{Na}_{2} \mathrm{O}, \mathrm{K}_{2} \mathrm{O}$ and $\mathrm{Al}_{2} \mathrm{O}_{3}$. The data for biotite-granite are well grouped except for $\mathrm{K}_{2} \mathrm{O}$ which shows the least amount of scatter. $\mathrm{SiO}_{2}$ is negatively correlated with $\mathrm{Al}_{2} \mathrm{O}_{3}, \mathrm{MgO}, \mathrm{Fe}_{2} \mathrm{O}_{3}, \mathrm{CaO}, \mathrm{TiO}_{2}$ and $\mathrm{P}_{2} \mathrm{O}_{5}$. All granitoids have high total alkali contents $\left(7 \%<\mathrm{Na}_{2} \mathrm{O}+\mathrm{K}_{2} \mathrm{O}<11 \%\right)$. They are K-rich, and transitional between the high-K calcalkaline and the shoshonitic series (Fig. 6). Their molar $\mathrm{A} / \mathrm{CNK}$ ratios vary between 0.93 and 1.09 except for samples CM2 and CM7. Thus, all rocks are metaluminous (Debon and Lemmet, 1999), mildly peraluminous and correspond to I-type granite After Chappell and White 
Table 4. Representative Analyses of Amphibole from Bapa-Batié Plutonic Rocks (wt\%). Number of Ions on the Basis of 23 Oxygens

\begin{tabular}{|c|c|c|c|c|c|c|c|c|c|c|c|c|c|c|c|c|c|c|c|}
\hline \multicolumn{12}{|c|}{ Granodiorite } & \multicolumn{8}{|c|}{ Biotite-Amphibole Granite } \\
\hline $\mathrm{N}^{\circ}$ & 1 & 2 & 3 & 4 & 5 & 6 & 7 & 8 & 9 & 10 & 11 & 12 & 13 & 14 & 15 & 16 & 17 & 18 & 19 \\
\hline $\mathrm{SiO}_{2}$ & 42.94 & 41.92 & 41.95 & 41.85 & 42.69 & 41.27 & 41.27 & 40.98 & 43.01 & 41.51 & 41.87 & 44.67 & 44.62 & 44.85 & 44.67 & 44.85 & 44.77 & 44.28 & 43.97 \\
\hline $\mathrm{Al}_{2} \mathrm{O}_{3}$ & 10.43 & 10.72 & 9.71 & 10.21 & 9.91 & 10.3 & 10.61 & 11.17 & 9.39 & 10.84 & 9.54 & 8.36 & 8.41 & 7.93 & 8.57 & 7.99 & 7.97 & 8.56 & 8.02 \\
\hline $\mathrm{TiO}_{2}$ & 0.52 & 0.72 & 0.42 & 0.59 & 0.66 & 0.72 & 0.44 & 0.44 & 0.52 & 0.49 & 0.55 & 1.03 & 0.85 & 0.77 & 0.89 & 0.97 & 0.75 & 0.93 & 0.91 \\
\hline $\mathrm{Fe}_{2} \mathrm{O}_{3}$ & 5.82 & 5.71 & 6.31 & 6.06 & 5.39 & 6.28 & 6.66 & 6.46 & 5.89 & 6.45 & 6.43 & 5.37 & 5.42 & 5.43 & 5.12 & 4.96 & 5.31 & 4.61 & 6.45 \\
\hline $\mathrm{FeO}$ & 16.33 & 16.18 & 17.21 & 16.54 & 16.74 & 16.68 & 15.25 & 15.91 & 15.25 & 15.72 & 15.26 & 13.49 & 13.45 & 13.53 & 13.35 & 13.94 & 13.41 & 14.01 & 12.91 \\
\hline $\mathrm{MgO}$ & 7.37 & 7.27 & 7.66 & 7.87 & 7.83 & 7.85 & 9.14 & 7.87 & 8.95 & 7.91 & 9.91 & 10.5 & 10.61 & 10.81 & 10.65 & 10.75 & 10.88 & 10.98 & 11.12 \\
\hline $\mathrm{CaO}$ & 11.33 & 11.95 & 11.51 & 11.59 & 11.56 & 11.71 & 11.61 & 11.67 & 11.65 & 11.67 & 11.69 & 11.61 & 11.65 & 11.61 & 11.82 & 11.65 & 11.69 & 11.51 & 11.53 \\
\hline $\mathrm{Na}_{2} \mathrm{O}$ & 1.37 & 1.36 & 1.21 & 1.24 & 1.39 & 1.28 & 1.57 & 1.39 & 1.36 & 1.41 & 1.34 & 1.46 & 1.34 & 1.39 & 1.28 & 1.47 & 1.28 & 1.55 & 1.51 \\
\hline $\mathrm{K}_{2} \mathrm{O}$ & 1.42 & 1.41 & 1.39 & 1.33 & 1.38 & 1.36 & 1.29 & 1.49 & 1.13 & 1.37 & 1.17 & 1.03 & 1.04 & 1.03 & 1.01 & 0.95 & 0.91 & 1.08 & 1.03 \\
\hline $\mathrm{MnO}$ & 0.49 & 0.44 & 0.44 & 0.87 & 0.49 & 0.53 & 0.41 & 0.41 & 0.56 & 0.81 & 0.35 & 0.54 & 0.46 & 0.51 & 0.51 & 0.49 & 0.45 & 0.45 & 0.48 \\
\hline $\mathrm{Cr}_{2} \mathrm{O}_{3}$ & 0.05 & 0.02 & 0.06 & 0.04 & 0.04 & 0.04 & 0.06 & 0.06 & 0.13 & 0.07 & 0.14 & 0.03 & 0.03 & 0.02 & 0.05 & 0.04 & 0.05 & 0.05 & 0.05 \\
\hline Total & 98.07 & 97.7 & 97.87 & 98.19 & 98.08 & 98.02 & 98.31 & 97.85 & 97.84 & 98.25 & 98.25 & 98.09 & 97.88 & 97.88 & 97.92 & 98.06 & 97.47 & 98.01 & 97.98 \\
\hline $\mathrm{Si}$ & 6.8154 & 6.8171 & 6.7829 & 6.7715 & 6.775 & 6.7716 & 6.7641 & 6.8081 & 6.7626 & 6.7974 & 6.7199 & 6.715 & 6.7217 & 6.7128 & 6.7184 & 6.6949 & 6.7141 & 6.6847 & 6.7075 \\
\hline AlIV & 1.1846 & 1.1829 & 1.2171 & 1.2285 & 1.225 & 1.2284 & 1.2359 & 1.1919 & 1.2374 & 1.2026 & 1.2801 & 1.285 & 1.2783 & 1.2872 & 1.2816 & 1.3051 & 1.2859 & 1.3153 & 1.2925 \\
\hline AlVI & 2.0903 & 2.0928 & 2.0422 & 2.0253 & 2.0306 & 2.0256 & 2.0144 & 2.0796 & 2.0122 & 2.0637 & 1.949 & 1.9417 & 1.9516 & 1.9385 & 1.9467 & 1.9119 & 1.9404 & 1.8969 & 1.9306 \\
\hline $\mathrm{Ti}$ & 0.0436 & 0.0604 & 0.0351 & 0.0492 & 0.055 & 0.06 & 0.0366 & 0.0369 & 0.0433 & 0.041 & 0.0455 & 0.0851 & 0.0703 & 0.0636 & 0.0736 & 0.0799 & 0.062 & 0.0765 & 0.0751 \\
\hline $\mathrm{Fe}^{3+}$ & 0.4882 & 0.4791 & 0.5268 & 0.505 & 0.4494 & 0.5234 & 0.5544 & 0.5413 & 0.4902 & 0.5396 & 0.5318 & 0.4438 & 0.4484 & 0.4486 & 0.4234 & 0.4087 & 0.4388 & 0.3793 & 0.5325 \\
\hline $\mathrm{Fe}^{2+}$ & 1.522 & 1.5084 & 1.5963 & 1.5316 & 1.5509 & 1.5446 & 1.4106 & 1.4812 & 1.4103 & 1.4612 & 1.4023 & 1.2387 & 1.2363 & 1.242 & 1.2265 & 1.2762 & 1.2312 & 1.2807 & 1.1842 \\
\hline $\mathrm{Mg}$ & 1.2266 & 1.2102 & 1.2688 & 1.3014 & 1.2954 & 1.2981 & 1.5097 & 1.3084 & 1.478 & 1.313 & 1.6262 & 1.7218 & 1.7415 & 1.772 & 1.7472 & 1.7575 & 1.7838 & 1.7924 & 1.8214 \\
\hline $\mathrm{Ca}$ & 0.5875 & 0.5877 & 0.5847 & 0.5837 & 0.584 & 0.5837 & 0.5831 & 0.5869 & 0.583 & 0.586 & 0.5793 & 0.5789 & 0.5794 & 0.5787 & 0.5792 & 0.5771 & 0.5788 & 0.5763 & 0.5782 \\
\hline $\mathrm{Na}$ & 1.9114 & 1.9119 & 1.9023 & 1.8991 & 1.9001 & 1.8991 & 1.897 & 1.9094 & 1.8966 & 1.9063 & 1.8846 & 1.8832 & 1.8851 & 1.8826 & 1.8842 & 1.8776 & 1.883 & 1.8747 & 1.8811 \\
\hline $\mathrm{K}$ & 0.0499 & 0.0499 & 0.0496 & 0.0495 & 0.0496 & 0.0495 & 0.0495 & 0.0498 & 0.0495 & 0.0497 & 0.0492 & 0.0491 & 0.0492 & 0.0491 & 0.0492 & 0.049 & 0.0491 & 0.0489 & 0.0491 \\
\hline $\mathrm{Mn}$ & 0.0463 & 0.0416 & 0.0414 & 0.0817 & 0.046 & 0.0498 & 0.0385 & 0.0387 & 0.0525 & 0.0764 & 0.0326 & 0.0503 & 0.0429 & 0.0475 & 0.0475 & 0.0455 & 0.0419 & 0.0417 & 0.0446 \\
\hline $\mathrm{Cr}$ & 0.0044 & 0.0018 & 0.0053 & 0.0035 & 0.0035 & 0.0035 & 0.0053 & 0.0053 & 0.0114 & 0.0062 & 0.0122 & 0.0026 & 0.0026 & 0.0017 & 0.0044 & 0.0035 & 0.0043 & 0.0043 & 0.0043 \\
\hline $\mathrm{Al}$ & 3.2749 & 3.2758 & 3.2593 & 3.2538 & 3.2556 & 3.2539 & 3.2503 & 3.2715 & 3.2496 & 3.2663 & 3.2291 & 3.2267 & 3.2299 & 3.2257 & 3.2283 & 3.217 & 3.2263 & 3.2122 & 3.2231 \\
\hline \multicolumn{20}{|c|}{ Name and classification after Leake et al. (1997) } \\
\hline$(\mathrm{Ca}) \mathrm{B}$ & 0.5875 & 0.5877 & 0.5847 & 0.5837 & 0.584 & 0.5837 & 0.5831 & 0.5869 & 0.583 & 0.586 & 0.5793 & 0.5789 & 0.5794 & 0.5787 & 0.5792 & 0.5771 & 0.5788 & 0.5763 & 0.5782 \\
\hline (Na)B & 1.4125 & 1.4123 & 1.4153 & 1.4163 & 1.416 & 1.4163 & 1.4169 & 1.4131 & 1.417 & 1.414 & 1.4207 & 1.4211 & 1.4206 & 1.4213 & 1.4208 & 1.4229 & 1.4212 & 1.4237 & 1.4218 \\
\hline$(\mathrm{Ca}+\mathrm{Na}) \mathrm{B}$ & 2 & 2 & 2 & 2 & 2 & 2 & 2 & 2 & 2 & 2 & 2 & 2 & 2 & 2 & 2 & 2 & 2 & 2 & 2 \\
\hline (Na)A & 0.4989 & 0.4995 & 0.487 & 0.4828 & 0.4841 & 0.4829 & 0.4801 & 0.4963 & 0.4795 & 0.4923 & 0.4639 & 0.4621 & 0.4645 & 0.4613 & 0.4633 & 0.4547 & 0.4618 & 0.451 & 0.4594 \\
\hline$(\mathrm{K}) \mathrm{A}$ & 0.0499 & 0.0499 & 0.0496 & 0.0495 & 0.0496 & 0.0495 & 0.0495 & 0.0498 & 0.0495 & 0.0497 & 0.0492 & 0.0491 & 0.0492 & 0.0491 & 0.0492 & 0.049 & 0.0491 & 0.0489 & 0.0491 \\
\hline$(\mathrm{Na}+\mathrm{K}) \mathrm{A}$ & 0.5488 & 0.5494 & 0.5366 & 0.5323 & 0.5337 & 0.5324 & 0.5296 & 0.5461 & 0.529 & 0.542 & 0.5131 & 0.5112 & 0.5137 & 0.5104 & 0.5125 & 0.5037 & 0.5109 & 0.4999 & 0.5084 \\
\hline $\mathrm{Mg} /\left(\mathrm{Mg}+\mathrm{Fe}^{2+}\right)$ & 0.4463 & 0.4452 & 0.4428 & 0.4594 & 0.4551 & 0.4566 & 0.517 & 0.469 & 0.5117 & 0.4733 & 0.537 & 0.5816 & 0.5848 & 0.5879 & 0.5876 & 0.5793 & 0.5916 & 0.5832 & 0.606 \\
\hline & Katop & $\begin{array}{r}\text { Katop } \\
\text { K }\end{array}$ & $\begin{array}{c}\text { Katop } \\
\text { atop }=\mathrm{K}\end{array}$ & $\begin{array}{l}\text { Katop } \\
\text { Satophori }\end{array}$ & $\begin{array}{l}\text { Katop } \\
\text { ite }\end{array}$ & Katop & MgKatop & Katop & MgKatop & Katop & MgKatop & MgKatop & MgKatop & MgKatop & MgKatop & MgKatop & MgKatop & MgKatop & MgKatop \\
\hline
\end{tabular}

(1974). The high $\mathrm{K}_{2} \mathrm{O}$ contents of the biotite-granite (3.93$6.63 \%)$, biotite-amphibole-granite (3.92-6.01\%), granodiorite (5.23-6.25) and fine-grained granite (5.8 to $6.63 \%)$ are especially worth noting. All the rocks form a ferriferous type association (Fe* $>0.73$; Fig. 7; [32, 33]).

\section{Trace Elements}

Trace element concentrations of the Bapa-Batié rocks are listed in Table 5. Selected elements are plotted against $\mathrm{SiO}_{2}$ content in Fig. (8). Ba, Sr, Y, Zr, and Co concentrations decrease with increasing $\mathrm{SiO}_{2}$ content. $\mathrm{Cr}, \mathrm{Ni}, \mathrm{Zn}$ and $\mathrm{Rb}$ concentrations are in general scattered on variation diagrams. $\mathrm{Ba} / \mathrm{Sr}$ ratios range between 2.5-5.9 and $\mathrm{Ba} / \mathrm{Rb}$ between 0.918.3; $\mathrm{Zr} / \mathrm{Nb}$ and $\mathrm{Zr} / \mathrm{Y}$ ratios are between $3-47.4$ and 8.2 to 40.4 respectively. These values are similar to those observed in continental calc-alkaline igneous suites (e.g. [34, 35]). Some features are noteworthy. The plutonic rocks of BapaBatié complex show a large scatter of data for $\mathrm{Ba}$ (3492739ppm), Cr (43-229ppm), Nb (5-30ppm), Rb (98$784 \mathrm{ppm})$ and $\mathrm{Sr}(98-609 \mathrm{ppm})$. Ba abundance is high in the granodiorite (762-2739 ppm) and moderate in biotiteamphibole-granite (565-1140 ppm).
Total REE concentrations are higher (179-533ppm) in biotite-granite and fine-grained granite (377-640ppm) compared to the biotite-amphibole-granite (127-269ppm) and the granodiorite (136-303ppm). Chondrite-normalized rare earth element (REE) patterns of the Bapa-Batié suite (Fig. 9) resemble each other, in general with a strong LREE enrichment with $\mathrm{La}_{N} / \mathrm{Yb}_{\mathrm{N}}$ ratios of 8-80 and $\mathrm{Gd}_{N} / \mathrm{Yb}_{\mathrm{N}}$ ratios of 1-10. Negative Eu* anomalies are pronounced in the biotite-granite $\left(\mathrm{Eu} / \mathrm{Eu}^{*}=0.25-0.46\right)$ and the biotiteamphibole-granite $\left(\mathrm{Eu} / \mathrm{Eu}^{*}=0.42-0.58\right)$ whereas they are not significant in the granodiorite $\left(\mathrm{Eu} / \mathrm{Eu}^{*}=0.79-1.05\right)$, and moderate $\left(\mathrm{Eu} / \mathrm{Eu}^{*}=0.96-1.0\right)$ to positive $\left(\mathrm{Eu} / \mathrm{Eu}^{*}=1.08\right.$ 1.32) in the fine-grained granite, indicating enrichment of europium relative to the neighbouring REE (Fig. 9g). The multi-element spectra (Fig. $\mathbf{9 b}, \mathbf{d}, \mathbf{f}, \mathbf{h}$ ) of all these rocks show a distinctive depletion in $\mathrm{Nb}, \mathrm{Sr}, \mathrm{P}, \mathrm{Ti}$ and $\mathrm{Y}$ relative to other trace elements; they are enriched in LILE (large ion lithophile elements) and display negative anomalies in $\mathrm{Sr}, \mathrm{Ba}$ and Ti. Distinctively higher contents of Th are observed in the biotite- granite and fine-grained granite. These rocks display less pronounced negative anomalies in $\mathrm{Sr}$ and $\mathrm{Ti}$, and lower $\mathrm{Rb}, \mathrm{Y}$ and $\mathrm{Yb}$ values resulting in more "fractionated" trace element distribution patterns, characteristic of calcalkaline arc granitoids. Overall, the enrichment in the 
Table 5. Representative Chemical Analyses of Bapa-Batié Plutonic Rocks (wt\%)

\begin{tabular}{|c|c|c|c|c|c|c|c|c|c|c|c|c|c|c|c|c|c|c|c|c|c|c|c|c|c|c|c|c|c|c|c|c|c|c|}
\hline Sample & CM1 & $\mathrm{CM} 2$ & CM3 & CM5 & CM6 & CM7 & $B$ irit & $\mathrm{CM}$ & CM11 & M12 & B4 & B5 & B7 & B9 & B11 & B13 & B15 & B16 & CM8 & B1 & B3 & B10 & B14 & B18 & \multirow{2}{*}{\multicolumn{5}{|c|}{$\begin{array}{ccc}\text { M1 } & \text { M1b } & \text { M3 } \\
\text { Quartz Syenites } \\
\end{array}$}} & \multirow{2}{*}{\multicolumn{5}{|c|}{ Fo 1 ine-Grained Granites }} \\
\hline $\begin{array}{l}\text { Rocks } \\
\end{array}$ & & & & & & Bioti & tite Grani & & & & & & & & & & & & & Biotite- & mphibole & Granites & & & & & & & & & & & & \\
\hline $\mathrm{SiO}_{2}$ & 72.74 & & 71.48 & 69.97 & & & & & 7088 & 69.61 & 70.76 & 70.5 & 71.46 & 72.8 & 72.82 & 72.41 & 68.87 & 69.73 & 74.74 & 70.59 & & 71.69 & 70.23 & 71.93 & 66.88 & & & & 65.1 & 70.7 & 59.07 & & 71.05 & 72.16 \\
\hline & & & & & & & & & & & & & & & & & & & & & & & & & & 0.80 & 0.82 & 0.93 & 0.78 & 0.4 & 0.55 & 0.49 & 0.42 & 0.4 \\
\hline & 13.74 & & 13.49 & 13.90 & & & & & & & & & & & & & & & 13.10 & & 14.31 & & & 14.22 & 15.23 & 15.52 & 15.68 & 16.12 & 16.83 & 14.33 & 15.2 & 14.55 & 14.33 & 13.78 \\
\hline $\begin{array}{l}\mathrm{Fe}_{2} \mathrm{He}_{2} \mathrm{O}_{3} \\
\end{array}$ & $\begin{array}{l}13.14 \\
2.17\end{array}$ & 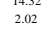 & $\begin{array}{l}13.49 \\
2.62\end{array}$ & $\begin{array}{l}13.90 \\
3.17\end{array}$ & 99 & $\begin{array}{l}1.70 \\
2.26\end{array}$ & $\begin{array}{l}1.64 \\
2.15\end{array}$ & $\begin{array}{l}13.64 \\
2.28\end{array}$ & $\begin{array}{l}14.53 \\
2.33\end{array}$ & $\begin{array}{l}1.51 \\
1.87\end{array}$ & $\begin{array}{l}15.12 \\
2.51\end{array}$ & $\begin{array}{l}1.244 \\
2.74\end{array}$ & $\begin{array}{l}1.84 \\
2.79\end{array}$ & $\begin{array}{l}1.84 \\
1.95\end{array}$ & $\begin{array}{l}13.72 \\
2.71\end{array}$ & 24 & $\begin{array}{l}16.21 \\
2.55\end{array}$ & $\begin{array}{l}14.07 \\
2.85\end{array}$ & $\begin{array}{l}15.100 \\
1.73\end{array}$ & $\begin{array}{l}14.57 \\
2.69\end{array}$ & $\begin{array}{l}1.31 \\
2.41\end{array}$ & $\begin{array}{l}14.21 \\
2.41\end{array}$ & $\begin{array}{l}15.12 \\
2.21\end{array}$ & $\begin{array}{l}1.222 \\
2.8\end{array}$ & $\begin{array}{l}15.23 \\
3.51\end{array}$ & $\begin{array}{l}15.52 \\
3.93\end{array}$ & $\begin{array}{l}15.68 \\
4.50\end{array}$ & $\begin{array}{l}16.12 \\
4.97\end{array}$ & $\begin{array}{l}1.683 \\
3.59\end{array}$ & $\begin{array}{l}1.3 .33 \\
2.64\end{array}$ & $\begin{array}{l}15.2 \\
3.84\end{array}$ & $\begin{array}{l}1.435 \\
3.29\end{array}$ & $\begin{array}{l}1.3 .35 \\
3.06\end{array}$ & $\begin{array}{l}1.837 \\
2.81\end{array}$ \\
\hline MnO & $\begin{array}{l}.2 .17 \\
0.04\end{array}$ & $\begin{array}{l}0.02 \\
0.03\end{array}$ & $\begin{array}{l}.202 \\
0.04\end{array}$ & $\begin{array}{l}3.17 \\
0.06\end{array}$ & & $\begin{array}{l}2.26 \\
0.03\end{array}$ & $\begin{array}{l}2.15 \\
0.06\end{array}$ & $\begin{array}{l}0.28 \\
0.05\end{array}$ & $\begin{array}{l}0.33 \\
0.05\end{array}$ & 0.04 & $\begin{array}{l}0.01 \\
0.03\end{array}$ & $\begin{array}{l}2.14 \\
0.04\end{array}$ & 0.03 & $\begin{array}{l}0.95 \\
0.03\end{array}$ & 0.03 & $\begin{array}{l}0.24 \\
0.03\end{array}$ & 0.02 & $\begin{array}{l}0.08 \\
0.03\end{array}$ & 0.06 & $\begin{array}{l}2.09 \\
0.01\end{array}$ & $\begin{array}{l}2.41 \\
0.04\end{array}$ & $\begin{array}{l}.241 \\
0.04\end{array}$ & $\begin{array}{l}0.21 \\
0.04\end{array}$ & $\begin{array}{l}2.8 \\
0.05\end{array}$ & 0.06 & $\begin{array}{l}0.93 \\
0.05\end{array}$ & $\begin{array}{l}x_{0.50} \\
0.06\end{array}$ & $\begin{array}{l}4.97 \\
0.10\end{array}$ & $\begin{array}{l}3.59 \\
0.09\end{array}$ & $\begin{array}{l}2.04 \\
0.03\end{array}$ & $\begin{array}{l}3.84 \\
0.06\end{array}$ & $\begin{array}{l}3.29 \\
0.03\end{array}$ & $\begin{array}{l}3.00 \\
0.05\end{array}$ & $\begin{array}{l}2.81 \\
0.03\end{array}$ \\
\hline $\mathrm{MgO}$ & 0.43 & 0.39 & $\begin{array}{l}0.58 \\
0.58\end{array}$ & $\begin{array}{l}0.606 \\
0.62\end{array}$ & 0.59 & 0.52 & 0.42 & 0.44 & 0.43 & 0.35 & 0.31 & 0.0 .36 & 0.03 & 0.03 & 0.03 & 0.03 & $\begin{array}{l}0.022 \\
0.02\end{array}$ & 0.03 & 0.19 & 0.24 & 0.25 & 0.04 & $\begin{array}{l}0.04 \\
0.04\end{array}$ & 0.05 & $\begin{array}{l}1.17 \\
17\end{array}$ & $\begin{array}{l}1.15 \\
\end{array}$ & $\begin{array}{l}1.48 \\
1.48\end{array}$ & $\begin{array}{l}1.63 \\
1.63\end{array}$ & $\begin{array}{l}0.92 \\
0.92\end{array}$ & 0.39 & 0.66 & 0.03 & 0.05 & 0.03 \\
\hline $\mathrm{CaO}$ & $\begin{array}{l}1.49 \\
1.49\end{array}$ & 1.11 & $\begin{array}{l}1.58 \\
\end{array}$ & $\begin{array}{l}1.62 \\
1.63\end{array}$ & $\begin{array}{l}1.65 \\
\end{array}$ & $\begin{array}{l}1.28 \\
\end{array}$ & $\begin{array}{l}1.42 \\
1.47\end{array}$ & $\begin{array}{l}1.53 \\
1.53\end{array}$ & $\begin{array}{l}1.44 \\
1.44\end{array}$ & $\begin{array}{l}1.33 \\
\text { S }\end{array}$ & $\begin{array}{l}1.2 \\
\end{array}$ & 1.28 & $\begin{array}{l}1.22 \\
1.22\end{array}$ & $\begin{array}{l}1.06 \\
1.06\end{array}$ & 1.38 & $\begin{array}{l}1.27 \\
1.27\end{array}$ & $\begin{array}{l}0.02 \\
1.2\end{array}$ & 1.51 & $\begin{array}{l}1.319 \\
1.31\end{array}$ & $\begin{array}{l}1.11 \\
11\end{array}$ & $\begin{array}{l}1.14 \\
1.14\end{array}$ & $\begin{array}{l}1.24 \\
1.21\end{array}$ & 2.18 & $\begin{array}{l}1.25 \\
1.25\end{array}$ & $\begin{array}{l}2.31 \\
2.39\end{array}$ & 2.12 & $\begin{array}{l}2.45 \\
2.52\end{array}$ & 2.88 & $\begin{array}{l}1.82 \\
1.82\end{array}$ & 1.41 & $\begin{array}{l}1.790 \\
1.79\end{array}$ & 1.51 & 1.23 & 1.32 \\
\hline $\mathrm{Na}_{2} \mathrm{O}$ & 3.87 & 3.02 & 3.51 & 2.97 & $\begin{array}{l}3.04 \\
\end{array}$ & $\begin{array}{l}3.00 \\
3.01\end{array}$ & 4.58 & 2.93 & 2.87 & $\begin{array}{l}3.00 \\
3.0\end{array}$ & 3.73 & $\begin{array}{l}3.71 \\
\end{array}$ & $\begin{array}{l}3.18 \\
\end{array}$ & 3.26 & $\begin{array}{l}3.41 \\
\end{array}$ & 3.46 & 3.66 & 3.13 & 3.81 & 3.6 & 3.58 & 3.78 & $\begin{array}{l}3.66 \\
3.64\end{array}$ & 3.81 & 3.49 & 3.48 & 3.52 & $\begin{array}{l}2.06 \\
4.09\end{array}$ & $\begin{array}{l}4.62 \\
4.24\end{array}$ & $\begin{array}{l}3.29 \\
\end{array}$ & 3.88 & 3.32 & 3.62 & 3.48 \\
\hline $\mathrm{K}_{2} \mathrm{O}$ & 3.93 & 4.51 & 4.45 & 5.63 & 5.57 & 4.86 & 4.45 & 6.01 & 6.9 & 6.55 & 5.78 & 5.61 & 5.48 & 5.82 & 5.02 & 5.17 & 6.63 & 5.52 & 3.92 & 6.01 & 5.92 & 5.44 & 5.5 & 5.33 & 5.26 & 5.67 & 5.42 & 5.23 & 6.23 & 6.63 & 5.13 & 5.51 & 5.85 & 5.18 \\
\hline LI & 0. & 0.31 & 0.51 & 0.51 & 0. & 0.86 & 0.56 & 1.50 & 0.92 & 1.45 & 0.04 & 0.6 & 0.35 & 0.55 & 0.21 & 0.11 & 0.11 & 0.21 & 0.20 & 0.6 & 0.35 & 0.44 & 0.11 & 0.21 & .48 & 0.50 & 0.58 & 0.71 & 0.62 & 0.03 & 0.04 & 43 & 0.11 & 0.22 \\
\hline $\mathrm{P}_{2} \mathrm{O}_{5}$ & 0.13 & 0.13 & 0.15 & 0.19 & 0. & 0.10 & 0.13 & 0.12 & 0.14 & 0.11 & 0.18 & 0.13 & 0.13 & 0.1 & 0.11 & 13 & 0.16 & 0.17 & 0.05 & 0.1 & 0.1 & 12 & 0.1 & 0.12 & 0.18 & 0.26 & 30 & 0.32 & 0.21 & 0.16 & 0.22 & 15 & 0.15 & 0.11 \\
\hline Total & 99.21 & 99.04 & 98.83 & 99.19 & 99.09 & 100.85 & 99.04 & 99.91 & 100.01 & 99.16 & 100 & 99.61 & 99.9 & 99.78 & 99.83 & 100 & 99.81 & 97.79 & 99.27 & 99.83 & 99.9 & 99.67 & 99.43 & 100.14 & 99.17 & 99.23 & 99.06 & 99.18 & 100.43 & 100.01 & 100.44 & 99.71 & 99.92 & 99.52 \\
\hline $\mathrm{A} / \mathrm{CNK}$ & 1.0 & 1.2 & 1.0 & 1.0 & 1.0 & 1.2 & 1.0 & 1.0 & 1.0 & 1.0 & 1.0 & 1.0 & 1.1 & 1.0 & 1.0 & 1.1 & 1.1 & 1.0 & 1.0 & 1.0 & 1.0 & 1.0 & 0.9 & 1.0 & 1.0 & 1.0 & 1.0 & 0.9 & 1.0 & 0.9 & 1.0 & 1.0 & 1.0 & 1.0 \\
\hline Rb & 251 & 255 & 258 & 265 & 258 & 210 & 362 & 302 & 784 & 412 & 356 & 268 & 312 & 339 & 224 & 331 & 363 & 211 & 177 & 294 & 320 & 330 & 121 & 336 & 9 & 144 & 150 & 150 & 129 & 251 & 287 & 279 & 331 & 218 \\
\hline & & & & & & & & & & & & & & & & & & & & & & & & & & & & & & & & & & $\begin{array}{l}150 \\
150\end{array}$ \\
\hline Ba & $\begin{array}{l}122 \\
349\end{array}$ & $\begin{array}{l}110 \\
443\end{array}$ & $\begin{array}{l}156 \\
538\end{array}$ & 707 & $\begin{array}{l}216 \\
708\end{array}$ & $\begin{array}{r}98 \\
433\end{array}$ & 321 & $\begin{array}{l}530 \\
534\end{array}$ & $\begin{array}{l}680 \\
687\end{array}$ & $\begin{array}{l}763 \\
756\end{array}$ & $\begin{array}{l}152 \\
814\end{array}$ & $\begin{array}{l}102 \\
666\end{array}$ & $\begin{array}{l}170 \\
627\end{array}$ & $\begin{array}{l}541 \\
541\end{array}$ & $\begin{array}{l}130 \\
496\end{array}$ & $\begin{array}{l}560 \\
565\end{array}$ & 853 & $\begin{array}{l}786 \\
765\end{array}$ & $\begin{array}{l}152 \\
897\end{array}$ & $\begin{array}{l}630 \\
634\end{array}$ & $\begin{array}{l}126 \\
565\end{array}$ & $\begin{array}{l}113 \\
592\end{array}$ & $\begin{array}{c}100 \\
1140\end{array}$ & $\begin{array}{l}601 \\
609\end{array}$ & $\begin{array}{l}709 \\
762\end{array}$ & $\begin{array}{l}\text { 96 } \\
1737\end{array}$ & $\begin{array}{l}1659 \\
1659\end{array}$ & $\begin{array}{l}6739 \\
2739\end{array}$ & $\begin{array}{r}2074 \\
2039\end{array}$ & $\begin{array}{l}198 \\
894\end{array}$ & $\begin{array}{l}180 \\
671\end{array}$ & 733 & $\begin{array}{l}171 \\
696\end{array}$ & 562 \\
\hline $\begin{array}{c}\text { Ba } \\
\text { Y }\end{array}$ & $\begin{array}{l}309 \\
20\end{array}$ & $\begin{array}{l}443 \\
23\end{array}$ & $\begin{array}{l}338 \\
16\end{array}$ & $\begin{array}{l}707 \\
22\end{array}$ & $\begin{array}{l}798 \\
19\end{array}$ & 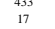 & $\begin{array}{l}321 \\
12\end{array}$ & $\begin{array}{l}334 \\
18\end{array}$ & $\begin{array}{l}687 \\
17\end{array}$ & $\begin{array}{l}556 \\
17\end{array}$ & $\begin{array}{l}814 \\
10\end{array}$ & $\begin{array}{c}666 \\
8\end{array}$ & $\begin{array}{l}627 \\
14\end{array}$ & $\begin{array}{l}541 \\
14\end{array}$ & & $\begin{array}{l}665 \\
11\end{array}$ & & $\begin{array}{c}765 \\
7\end{array}$ & & $\begin{array}{c}634 \\
5\end{array}$ & 565 & $\begin{array}{c}592 \\
8\end{array}$ & & $\begin{array}{l}6099 \\
10\end{array}$ & & & 599 & 39 & & $\begin{array}{c}894 \\
7\end{array}$ & $\begin{array}{c}671 \\
8\end{array}$ & $\begin{array}{l}723 \\
22\end{array}$ & $\begin{array}{l}656 \\
25\end{array}$ & $\begin{array}{c}562 \\
9 \\
\text { a }\end{array}$ \\
\hline $\begin{array}{l}\mathrm{Y} \\
\mathrm{Zr}\end{array}$ & $\begin{array}{l}20 \\
182\end{array}$ & $\begin{array}{l}23 \\
186 \\
18\end{array}$ & $\begin{array}{l}16 \\
222\end{array}$ & $\begin{array}{l}22 \\
315 \\
3\end{array}$ & $\begin{array}{l}19 \\
276\end{array}$ & $\begin{array}{l}17 \\
217\end{array}$ & $\begin{array}{l}12 \\
117\end{array}$ & $\begin{array}{l}18 \\
174\end{array}$ & $\begin{array}{l}17 \\
175\end{array}$ & $\begin{array}{l}17 \\
219\end{array}$ & $\begin{array}{l}10 \\
249\end{array}$ & $\begin{array}{c}8 \\
237 \\
237\end{array}$ & $\begin{array}{l}14 \\
224\end{array}$ & $\begin{array}{l}14 \\
161\end{array}$ & $\begin{array}{c}4 \\
218\end{array}$ & $\begin{array}{l}11 \\
164\end{array}$ & $\begin{array}{r}925 \\
225\end{array}$ & 283 & $\begin{array}{l}27 \\
117\end{array}$ & $\begin{array}{c}5 \\
115\end{array}$ & $\begin{array}{c}6 \\
173 \\
\end{array}$ & $\begin{array}{c}8 \\
179\end{array}$ & 111 & $\begin{array}{l}10 \\
156\end{array}$ & $\begin{array}{l}12 \\
302\end{array}$ & $\begin{array}{l}16 \\
287\end{array}$ & $\begin{array}{l}17 \\
276\end{array}$ & $\begin{array}{l}25 \\
283 \\
283\end{array}$ & $\begin{array}{l}29 \\
344\end{array}$ & 229 & $\begin{array}{c}8 \\
310 \\
3\end{array}$ & $\begin{array}{l}22 \\
298 \\
298\end{array}$ & $\begin{array}{l}25 \\
240\end{array}$ & 265 \\
\hline $\mathrm{v}$ & $\begin{array}{l}182 \\
17\end{array}$ & $\begin{array}{l}180 \\
20\end{array}$ & $\begin{array}{l}282 \\
28\end{array}$ & 27 & 27 & $\begin{array}{l}171 \\
19\end{array}$ & 18 & 22 & 26 & 22 & $\begin{array}{l}489 \\
18\end{array}$ & 18 & $\begin{array}{l}124 \\
17\end{array}$ & $\begin{array}{l}21 \\
21\end{array}$ & & $\begin{array}{l}204 \\
23\end{array}$ & 223 & 23 & 11 & 115 & & $1 / 9$ & 111 & & 302 & 45 & 52 & 64 & 344 & 229 & & 298 & & 263 \\
\hline $\mathrm{Nb}$ & 30 & 24 & 28 & 28 & 27 & 18 & 31 & 33 & 58 & 30 & 8 & 5 & 12 & 6 & 6 & 8 & $\begin{array}{l}1 \\
10\end{array}$ & 6 & 11 & 7 & 5 & 8 & 6 & 21 & 9 & 16 & 16 & 16 & 20 & 6 & 16 & 8 & 11 & 6 \\
\hline Cr & 70 & 115 & 63 & 81 & 71 & $\begin{array}{l}68 \\
68\end{array}$ & 102 & 131 & 229 & 116 & 125 & 158 & $\begin{array}{l}12 \\
191\end{array}$ & 178 & 171 & 165 & $\begin{array}{l}157 \\
157\end{array}$ & 49 & 61 & 134 & 122 & $\begin{array}{l}167 \\
167\end{array}$ & 156 & $\begin{array}{l}11 \\
120\end{array}$ & 59 & 43 & 53 & $\begin{array}{l}58 \\
58\end{array}$ & 94 & 150 & 141 & 189 & 136 & 144 \\
\hline $\mathrm{Ni}$ & 3 & 2 & 4 & 7 & $\begin{array}{l}1 \\
4\end{array}$ & $\begin{array}{c}08 \\
3\end{array}$ & 4 & $\begin{array}{c}51 \\
5\end{array}$ & 229 & 14 & & 4 & 4 & 4 & 111 & 4 & (3) & 10 & $\begin{array}{l}11 \\
2\end{array}$ & $\begin{array}{l}454 \\
4\end{array}$ & 122 & $10 \%$ & & 21 & 4 & $\begin{array}{l}43 \\
8\end{array}$ & 53 & 9 & $\begin{array}{l}\frac{9}{4} \\
5\end{array}$ & 150 & 4 & 109 & 4 & 3 \\
\hline $\mathrm{Cu}$ & 23 & 5 & 11 & 6 & 5 & 6 & 8 & 9 & 15 & 10 & 6 & 7 & 6 & 7 & 8 & 8 & 9 & 7 & 5 & & & & & & 6 & 6 & 4 & 2 & 7 & & & & & \\
\hline $\mathrm{Zn}$ & 314 & 223 & 198 & 191 & 210 & $\begin{array}{l}197 \\
\end{array}$ & 271 & 142 & 171 & 141 & 158 & 154 & 170 & 143 & 144 & 145 & 157 & 151 & 140 & 32 & 44 & 49 & 25 & 48 & 34 & 42 & 48 & 63 & 155 & 49 & 80 & 57 & 54 & 43 \\
\hline Ga & 28 & 36 & 46 & 38 & 50 & 36 & 33 & 39 & 89 & 52 & 28 & 33 & 31 & 27 & 29 & 30 & 31 & 30 & 50 & & & & & & 45 & 70 & 70 & 102 & 105 & & & & & \\
\hline Be & 6 & 4 & 5 & 5 & 5 & 3 & 12 & 6 & 5 & 6 & 3 & 3 & 6 & 6 & 7 & 7 & 7 & 6 & 6 & & & & & & 2 & 3 & 3 & 5 & 3 & & & & & \\
\hline Sc & 5 & 5 & 6 & 5 & 5 & 5 & 6 & 6 & 12 & 5 & 6 & 6 & 3 & 4 & 5 & 5 & 7 & 5 & 6 & & & & & & 5 & 9 & 1 & 11 & 9 & & & & & \\
\hline Co & 3 & 3 & 4 & 4 & 4 & 4 & 3 & 3 & 3 & 3 & 2 & 3 & 3 & 2 & 2 & 4 & 3 & 4 & 1 & 1.18 & 1.21 & 1.16 & 1.112 & 1.1087 & 4 & 6 & 7 & & 5 & 5.21 & 5.198 & 6.921 & 5.456 & 5.209 \\
\hline La & & 65.837 & 84.1 & 110. & 100 & 86.472 & 33.325 & & & & & & & & & 78.7. & & & & & & & & & & & & & & & & & & \\
\hline Ce & & $\begin{array}{l}130.811 \\
130.51\end{array}$ & $\begin{array}{l}8.4272 \\
162.631\end{array}$ & $\begin{array}{l}221.627 \\
2211\end{array}$ & $\begin{array}{l}20.01 .182 \\
20189\end{array}$ & $\begin{array}{l}150.4716 \\
158.71\end{array}$ & 66.462 & $\begin{array}{l}152.467 \\
152.47\end{array}$ & 136.988 & 133.921 & $\begin{array}{l}137.211 \\
137.25\end{array}$ & 1778.010 & 200.270 & $\begin{array}{l}1177.190 \\
119\end{array}$ & 195. & 115.810 & & 151. & & & $\begin{array}{l}119.000 \\
119.00\end{array}$ & 00 & 000 & & 54.016 & 18.932 & 122.503 & & & & & & & $\begin{array}{l}278.0000 \\
27800\end{array}$ \\
\hline Pr & $\begin{array}{l}13.977 \\
\end{array}$ & 13.833 & 17.421 & 23.945 & 21.444 & 17.032 & 7.431 & $\begin{array}{l}16.527 \\
\end{array}$ & 17.153 & 14.275 & 15.132 & 16.311 & 16.010 & $\begin{array}{l}18.010 \\
\end{array}$ & & 21.070 & 22.312 & & & 36 & 8.560 & 23 & 82 & & & & $\begin{array}{l}12.917 \\
\end{array}$ & & & 0.220 & & & & 12.084 \\
\hline $\mathrm{Nd}$ & & & & & & & & & & & & & & & & & & & & & & & & & & & & & & & & & & \\
\hline $\begin{array}{l}\mathrm{Nd} \\
\mathrm{Sn}\end{array}$ & & & & & & & $\begin{array}{l}26.2733 \\
4.765\end{array}$ & & & & & & & & & & & & & & & & & & & & & & & & & & $\begin{array}{l}\begin{array}{l}44.000 \\
12.094\end{array}\end{array}$ & \\
\hline $\mathrm{E}$ & $\begin{array}{l}9.6018 \\
0.731\end{array}$ & & $\begin{array}{l}9.929 \\
0.790\end{array}$ & & & $\begin{array}{l}9.6034 \\
0.777\end{array}$ & 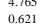 & $\begin{array}{l}9.6 .74 \\
0.811\end{array}$ & $\begin{array}{l}13.634 \\
1.127\end{array}$ & $\begin{array}{l}. .548 \\
0.938\end{array}$ & & & & & & & & & & & & & & & & & & & & & .2250 & $\begin{array}{l}11.982 \\
3.115\end{array}$ & $\begin{array}{l}32.094 \\
3.487\end{array}$ & $\begin{array}{l}32.736 \\
3.977\end{array}$ \\
\hline $\begin{array}{l}\mathrm{E} \\
\mathrm{G}\end{array}$ & & & $\begin{array}{l}5.070 \\
5.899\end{array}$ & 8.1 & $\begin{array}{l}1.163 \\
6.940\end{array}$ & $\begin{array}{l}5.7 \\
5.9\end{array}$ & & & & $\begin{array}{l}5.9 \\
5.9\end{array}$ & & & & & & & $\begin{array}{l}8.1 \\
8.0\end{array}$ & & & & & & & & & & & & & & & $\begin{array}{l}3.115 \\
8.234\end{array}$ & $\begin{array}{l}.3487 \\
8.008\end{array}$ & $\begin{array}{l}3.9971 \\
7.998\end{array}$ \\
\hline $\mathrm{T}$ & & & $\begin{array}{l}0.8999 \\
0.784\end{array}$ & $\begin{array}{l}8.1 \\
0.9\end{array}$ & $\begin{array}{l}\begin{array}{l}.9440 \\
0.828\end{array}\end{array}$ & 0.7 & & $\begin{array}{l}6.4 \\
0.8\end{array}$ & & $\begin{array}{l}3.9 \\
0.7\end{array}$ & & & & & & & & & & & & & & & & & & & & & & $\begin{array}{l}254 \\
9993\end{array}$ & $\begin{array}{l}8.008 \\
1.012\end{array}$ & $\begin{array}{l}1.988 \\
1.232\end{array}$ \\
\hline$D_{3}$ & & 4. & $\begin{array}{l}3.084 \\
3.843\end{array}$ & $\begin{array}{l}0.9 \\
4.7\end{array}$ & $\begin{array}{l}4.8 \\
4.2\end{array}$ & 3.8 & 2.1 & & & 3.8 & & & & & & & 4. & & & & & & & & & & & & & & & 002 & $\begin{array}{l}7.012 \\
7.262\end{array}$ & 835 \\
\hline $\mathrm{H}$ & & 0.8 & 0.5 & 0.7 & 0.6 & 0.5 & 0.3 & 0.6 & & & 0.6 & & 0.5 & & & & 0. & $\begin{array}{l}4.2 \\
0 .\end{array}$ & & & & & & & & 0. & & & & & 1.046 & 076 & $\begin{array}{l}1.1182 \\
1.18\end{array}$ & 198 \\
\hline $\mathrm{E}$ & & 1.8 & 1. & 1.9 & 1.7 & & 0.9 & 1.553 & & 1. & 1.4 & & 1.6 & & & & & 1. & & & & & & & & & & & & & & 984 & 2.874 & 833 \\
\hline $\mathrm{T}_{\mathrm{T}}$ & & 0.2 & 0.1 & 0.2 & 0.2 & 0.1 & 0.1 & 0.191 & & 0.216 & 0.1 & & 0.2 & & 0.2 & & & 0.2 & & & 0.1 & & & & & 0.19 & 0.239 & & & 0.243 & 0.350 & 344 & 0.354 & 0.367 \\
\hline $\mathbf{Y}$ & & 1.4 & 1.1 & 1.5 & & & & $\begin{array}{l}1.11 \\
\end{array}$ & & & & & 1. & & & & & & & & & & & & & & & & & & & & 2.978 & 2.998 \\
\hline L & & & & & & & & & & & & & & & & & & & & & & & & & & & & & & & & & 412 & \\
\hline H & & & & & & & & & & & & & & & & & & & & & & & & & & & & & & & & & & \\
\hline $\mathrm{T}$ & & & & & & & & & & & 2. & & & & & & & & & & & & & & & & & & & & & & & \\
\hline $\mathbf{P}$ & & & & & & & 24. & & & & 13. & & & & & & & & & & & & & & & & & & & & & & & \\
\hline T & & 44.983 & & & & & 24. & & & & 27. & & 41. & & & & & & & & & & & & & & & & & & & & & \\
\hline $\mathbf{u}$ & 4.406 & & 4.525 & 5.504 & 4.709 & 8.598 & 7.226 & 7.410 & 9.118 & 8.685 & 9.000 & 10.000 & 8.000 & 10.000 & 9.000 & 10.000 & 11.000 & 9.000 & 5.347 & 11.000 & 9.000 & 8.000 & 8.000 & 11.000 & 2.574 & 2.626 & 2.310 & 3.315 & 2.368 & 11.000 & 10.000 & 9.000 & 9.000 & 10.000 \\
\hline & & & & 49.395 & & 48.545 & & 46.531 & 62.242 & 41.8 & 42.2 & & 57.372 & & 68. & & & & 8.1283 & 14.552 & & & & & 17.075 & 31.056 & 27.189 & & & 37.741 & 33.277 & 37.107 & 34.678 & \\
\hline & & 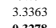 & & 4.3697 & & & & & & & & & & & & & & & & & & & & & & & & & & & 2.2882 & 2.3153 & 2.1731 & 2.1559 \\
\hline & & & & & & & & & & & & & & & & & & & & & & & & & & & & & & & & & & \\
\hline
\end{tabular}

incompatible elements $(\mathrm{K}, \mathrm{Rb}$, and $\mathrm{Ba})$ and depletion in high field strength elements (HFSE) supports the importance of the crustal source component in the genesis of the BapaBatié granitoids. Thus the overall distribution of major and trace elements observed is typical of those of calc-alkaline rocks.

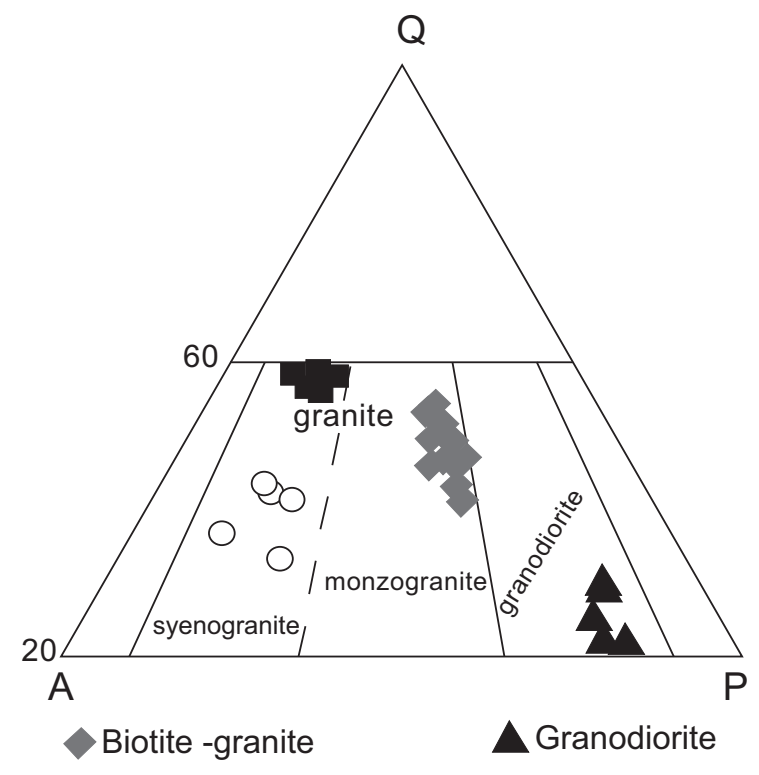

Biotite-amphibole granite OFine-grained granite

Fig. (4). Position of the Bapa-Batié granitoids in the QAPF diagram.

Summarily, the geochemical variations confirm the presence of four distinct types of plutonic rocks within the
Bapa-Batié area. These plutonic rocks are metaluminous, high-K calc-alkaline ferro-potassic in composition. They have petrographical and geochemical characteristics of Itype rocks. Each plutonic group is characterized by specific magmatic evolution trend on the geochemical variation diagrams.

In terms of major and trace elements, some features are common to all plutons of the Bapa-Batié, eastern Nigeria [36-38] and Borborema Province in the NE Brazil [39-43]: (1) the rocks are high-K calc-alkaline to shoshonitic associations (Fig. 10a); (2) they are also metaluminous to weakly peraluminous (Fig. 10b); (3) silica contents typically $>50 \mathrm{wt} \%$; (4) the enrichment in the incompatible elements $(\mathrm{K}, \mathrm{Rb}$, and $\mathrm{Ba})$ and depletion in high field strength elements (HFSE). Although one difference between the studied plutons, eastern Nigeria plutons and those of the Borborema Province in the NE Brazil are apparent in Fig. (10c), the Bapa-Batié and eastern Nigeria plutons form a ferriferous type association while the plutons of the Borborema Province in the NE Brazil are magnesian.

\section{Zirconium Saturation Geothermometry}

Pearce (1982) [44], Harrison and Watson (1983) [45], Watson and Harrison (1983) [46] demonstrated that the $\mathrm{Zr}$ contents of igneous rocks would be inherited from their source rocks and controlled by degree of partial melting involved during their generation; high $\mathrm{Zr}$ contents correspond to higher degrees of partial melting for the same source rock. Granitoids from the Bapa-Batié area could come from similar source rocks, and their $\mathrm{Zr}$ distributions could be ascribed to quite different degrees of partial melt of that source rock. 

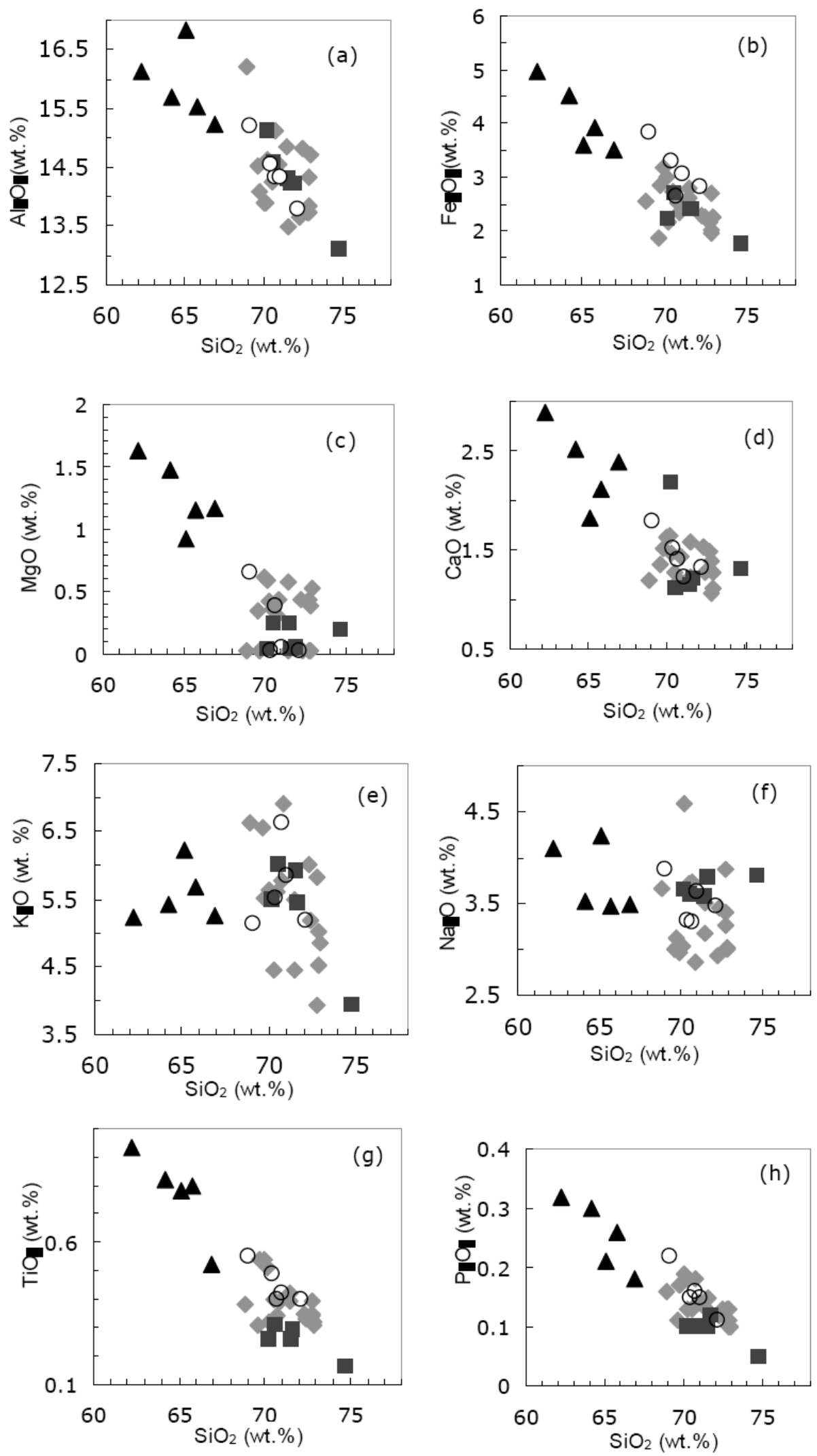

Fig. (5). Harker diagrams of selected major elements. Symbols as in Fig. (4).

We applied the $\mathrm{Zr}$ saturation geothermometer $[45,46]$ to the Bapa-Batie granitoids to estimate the minimum temperatures of the melts (assuming that there is no residual zircon inherited from the source). The calculated temperatures (Table 6) range between $749-839^{\circ} \mathrm{C}$ for the biotite granite, $741-789^{\circ} \mathrm{C}$ for the amphibole-biotite granite, $801-834^{\circ} \mathrm{C}$ for the granodiorite and $802-838^{\circ} \mathrm{C}$ for the finegrained granite. 
(a)

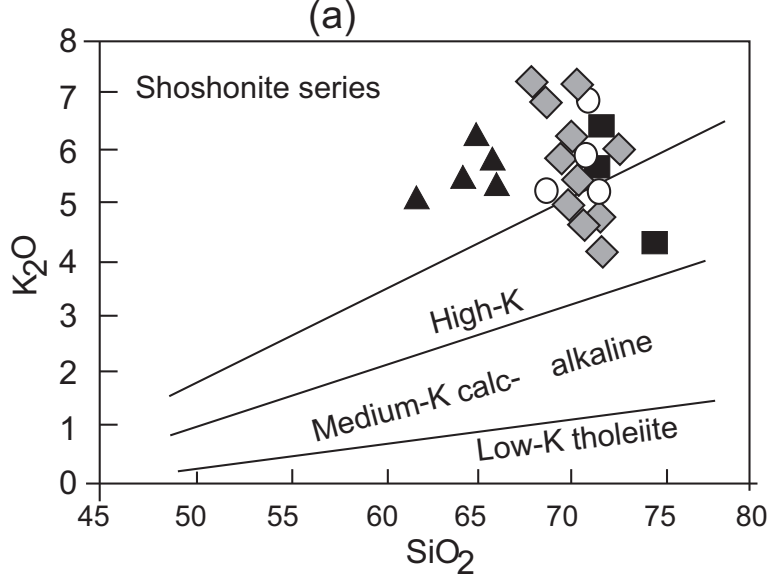

(b)

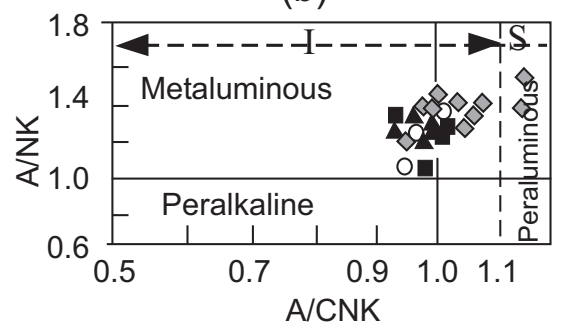

Fig. (6). (a) $\mathrm{SiO}_{2}$ vs $\mathrm{K}_{2} \mathrm{O}$ diagram showing high-K calc-alkaline and shoshonitic Affinity of the Bapa-Batié granitoids After [71, 72]: dotted line. (b) A/NK vs A/CNK diagram showing metaluminous weakly peraluminous character of the Bapa-Batié granitoids.

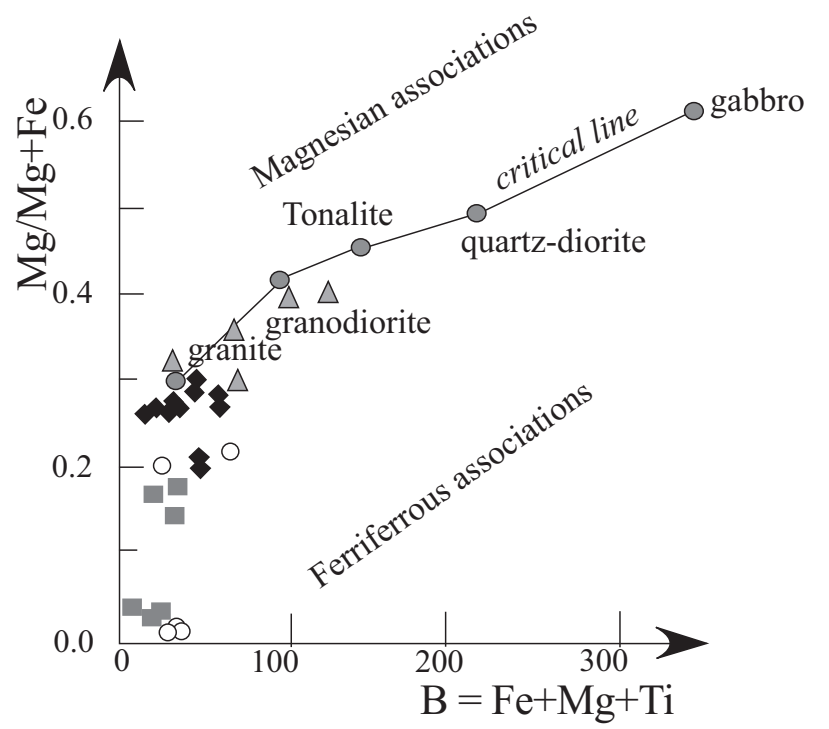

Fig. (7). $\mathrm{Mg} / \mathrm{Mg}+\mathrm{Fe}$ diagram of Debon and Lemmet (1999) [32]. Symbols as in Fig. (4).

\section{Zircon $U-P b$ Data}

The most common textural features include bands parallel to the prism faces oscillatory zoning, typical of growth in magmatic conditions. All analytical points are concordant and overlap nicely on the Concordia curve for each sample, using the software Isoplot/Ex [31] at $95 \%$ confidence level.
Zircon grains of biotite- granite (sample Batié) and biotite- amphibole granite (sample CM8) are elongated with euhedral to subhedral shape; oscillatory zoning, typical of magmatic growth, is common (Fig. 11). Tables 7 and $\mathbf{8}$ show the results of analytical data for the studied samples. The zircon grains plot close to Concordia (Fig. 12) at $600 \pm 3.7$ $\mathrm{Ma}(\mathrm{MSWD}=2.1)$ and $619 \pm 19 \mathrm{Ma}(\mathrm{MSWD}=0.23)$. The high concordance of zircon grains and the typical magmatic growth indicate that these ages of $600 \mathrm{Ma}$ and $619 \mathrm{Ma}$ are those of the crystallization of the zircons in the biotiteamphibole granite and biotite- granite, respectively, and also correspond to the age of emplacement during the PanAfrican orogeny.

\section{DISCUSSION}

\section{Constraints on the Source of the Bapa-Batié Plutonic Rocks}

The investigated plutonic rocks exhibit petrographical and chemical compositions characteristic of high-K to shoshonitic I-type granitoids [47, 48] derived from partial melting of igneous protoliths. From the several studies carried out to constrain the generation of high-K magmas in convergent tectonic settings, two main processes are commonly recognized [49]: (i) in continental arc settings, parent mantle melts that are enriched in slab-derived fluids may become contaminated with crustal material during ascent [50]; (ii) in syn- to post-collisional settings, crustal source rocks may occur as a consequence of decompression following delamination of the lithospheric root or slab breakoff (e.g. [51, 52]). The geochemical compositions of Bapa-Batié granitoids as shown on Harker's diagrams did not indicate continuous compositional variation from one group to another but little internal compositional variation and overlap between some groups. This suggests that the compositional variability of these granitoids does not appear to be generated mainly by fractionation processes but probably reflects the primary compositions of anatectic melts generated at various crustal levels. The nature of the igneous source can be constrained using the geochemical signatures of the plutonic rocks. The REE and multi-element patterns (Fig. 9) suggest genetic processes involving garnet. The high contents of LREE in all rocks could be related either to the enrichment of their source materials in LREE, or to a low degree of partial melting of source protoliths with garnet and amphibole among the residual phases. However, their concentrations in HREE (more or less 10 times the chondrite values) do not support the assumption of a source containing garnet. Moreover, the spider diagrams (Fig. 9) show negative anomaly in $\mathrm{Y}$, suggesting that garnet was involved in the residual phase. The spider diagrams characteristically display negative anomalies for $\mathrm{Ba}, \mathrm{Nb}, \mathrm{Sr}, \mathrm{P}, \mathrm{Ti}$ and $\mathrm{Y}$. As explained above, concentration of elements in a melt derived by partial melting depends upon both the content of these elements in the source and their retention in the residue during partial melting. The geochemistry and mineralogy of the granitic rocks reflect not only the nature of the protoliths from which they were derived, but also the dynamic conditions under which magmas were formed, evolved and eventually solidified [53]. Compositional differences of melts produced by partial melting of different source rocks, such as amphibolites, tonalitic gneisses, metapelites and 

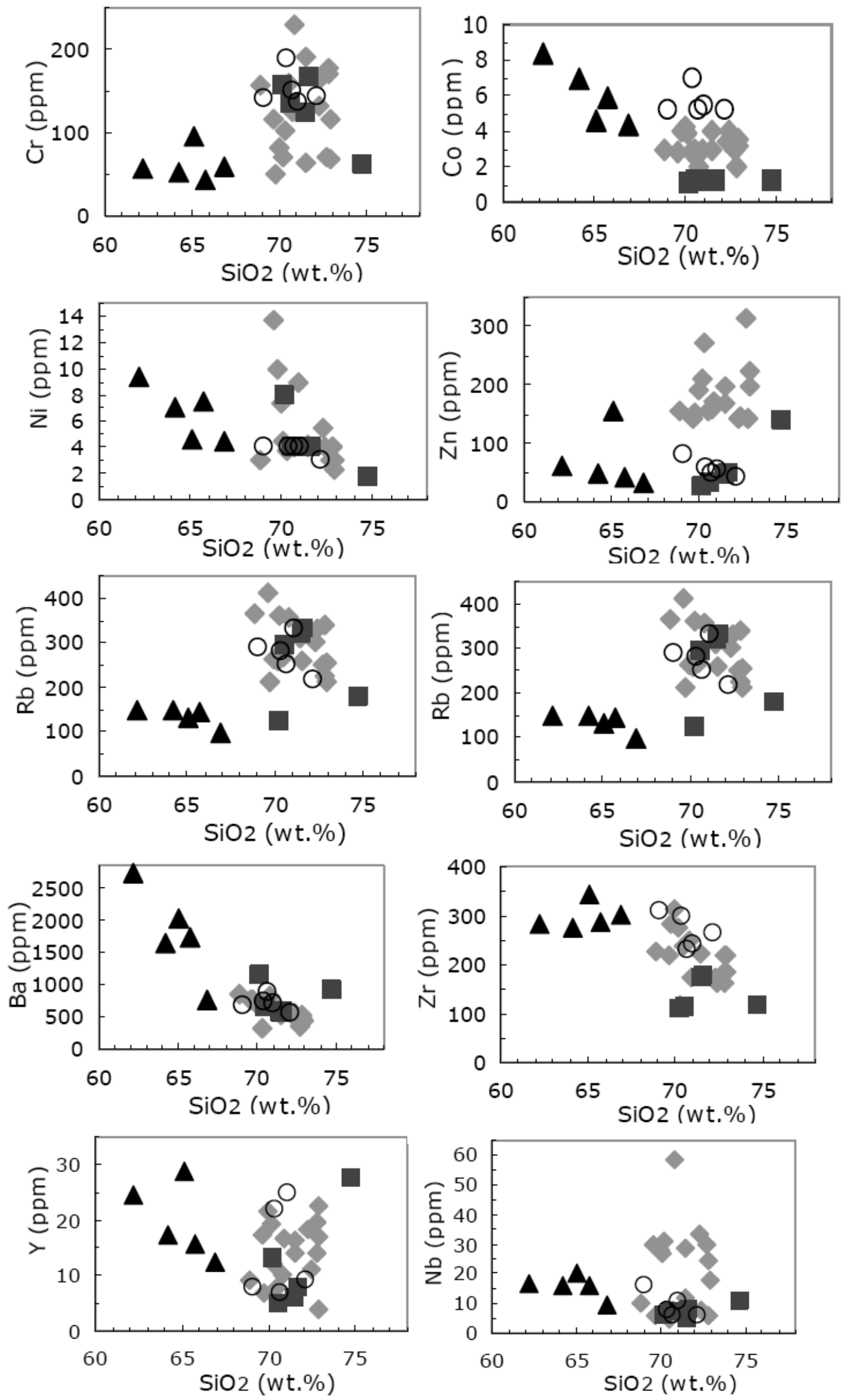

Fig. (8). Harker diagrams of selected trace elements. Symbols as in Fig. (4). 

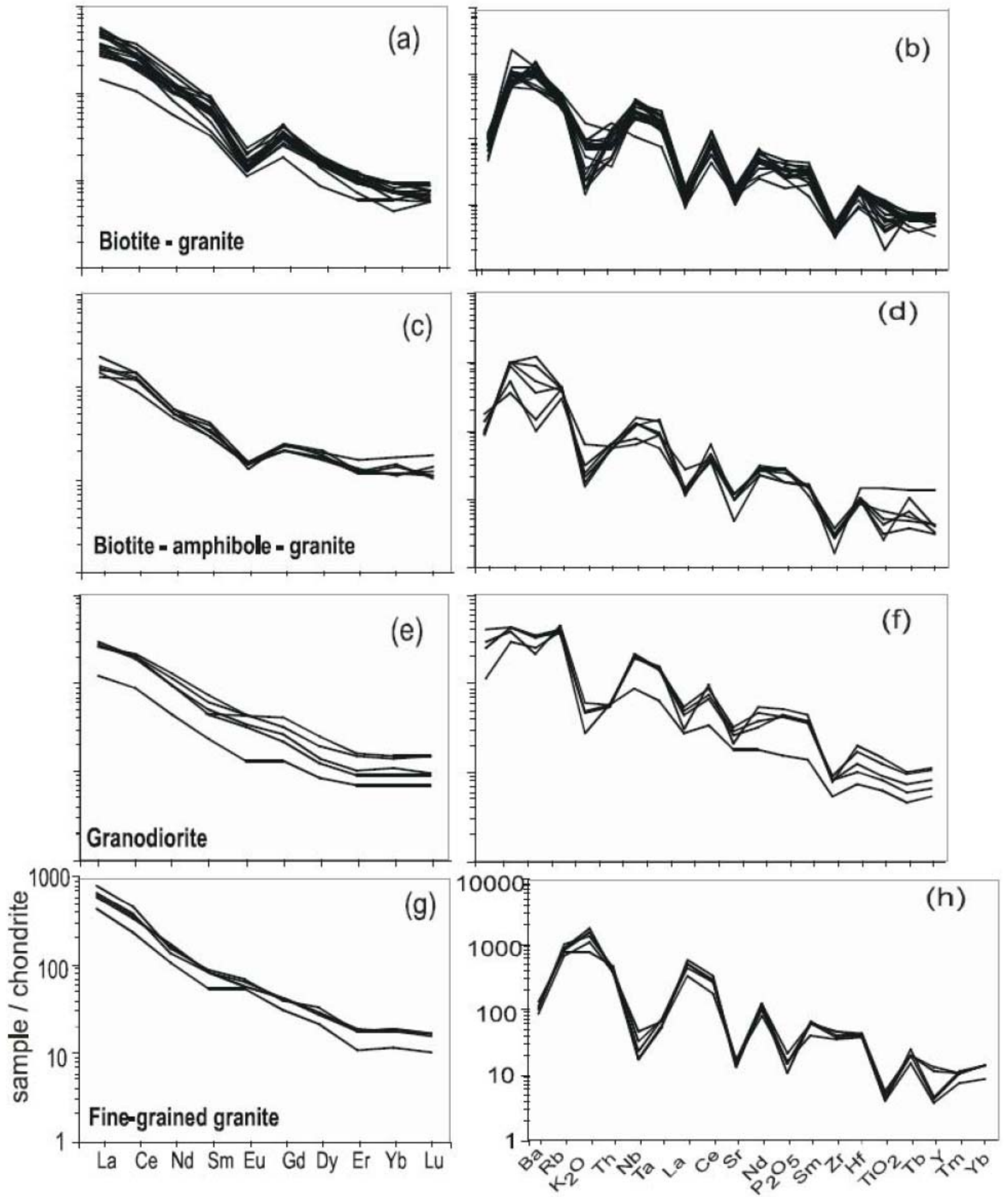

Fig. (9). Chondrite-normalised REE patterns for the Bapa-Batié granitoids. The normalizing values are from [73]. 
(a)

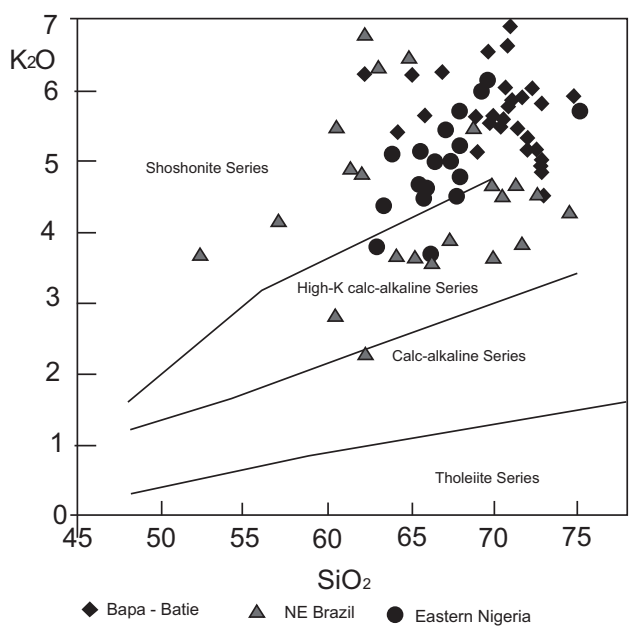

(b)

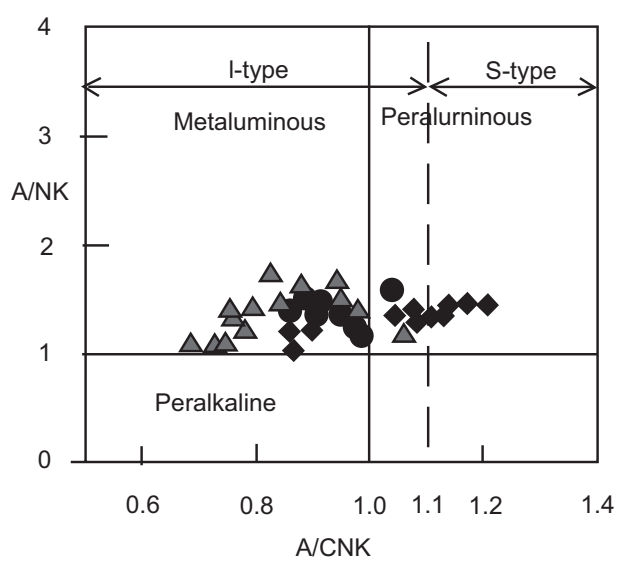

(c)

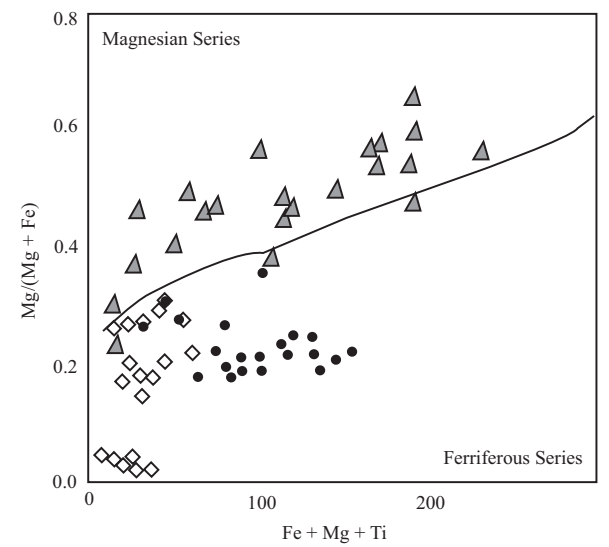

Fig. (10). (a) $\mathrm{SiO}_{2} v s \mathrm{~K}_{2} \mathrm{O}$ and (b) $\mathrm{A} / \mathrm{NK}$ vs $\mathrm{A} / \mathrm{CNK}$ diagrams showing the same high-K calc-alkaline and shoshonitic Affinity, metaluminous weakly peraluminous character of the Bapa-Batié granitoids, Borborema province in NE Brazil granitoids [40, 41] and eastern Nigeria granitoids [37, 38]. (c) Magnesian associations of NE Brazil granitoids different for those ferriferous of Bapa-Batié and eastern Nigeria granitoids.
Table 6. Estimate of the Minimum Temperature of the Melts of the Bapa-Batié Granitoids with the Zirconium Saturation Geothermometry $[45,46]$

\begin{tabular}{|c|c|c|}
\hline Sample & $\mathbf{Z r}(\mathbf{p p m})$ & Temperature $\left({ }^{\circ} \mathbf{C}\right)$ \\
\hline CM1 & 182 & 796 \\
\hline $\mathrm{CM} 2$ & 186 & 815 \\
\hline CM3 & 222 & 810 \\
\hline CM5 & 315 & 839 \\
\hline CM6 & 276 & 826 \\
\hline CM7 & 217 & 825 \\
\hline Batie & 117 & 749 \\
\hline CM11a & 174 & 784 \\
\hline CM11b & 175 & 784 \\
\hline CM12 & 219 & 806 \\
\hline B4 & 249 & 821 \\
\hline B5 & 237 & 811 \\
\hline B7 & 224 & 821 \\
\hline B9 & 161 & 784 \\
\hline B11 & 218 & 810 \\
\hline B13 & 164 & 792 \\
\hline B15 & 225 & 810 \\
\hline B16 & 283 & 832 \\
\hline CM8 & 117 & 760 \\
\hline B1 & 115 & 751 \\
\hline B3 & 173 & 785 \\
\hline B 10 & 179 & 789 \\
\hline B14 & 111 & 741 \\
\hline B18 & 156 & 777 \\
\hline $\mathrm{CM} 2 \mathrm{~b}$ & 302 & 825 \\
\hline M1 & 287 & 820 \\
\hline M1b & 276 & 811 \\
\hline M3 & 283 & 801 \\
\hline M5 & 344 & 834 \\
\hline B2 & 229 & 802 \\
\hline B6 & 310 & 834 \\
\hline B8 & 298 & 838 \\
\hline B12 & 240 & 813 \\
\hline B17 & 265 & 827 \\
\hline
\end{tabular}

metagreywackes, under variable melting conditions can be visualized in terms of molar $\mathrm{CaO} /\left(\mathrm{MgO}+\mathrm{FeO}_{\text {total }}\right)$ vs molar $\mathrm{Al}_{2} \mathrm{O}_{3} /\left(\mathrm{MgO}+\mathrm{FeO}_{\text {total }}\right)$ ([49]; Fig. 13). In that diagram, most of the samples plot in the field of partial melts from 

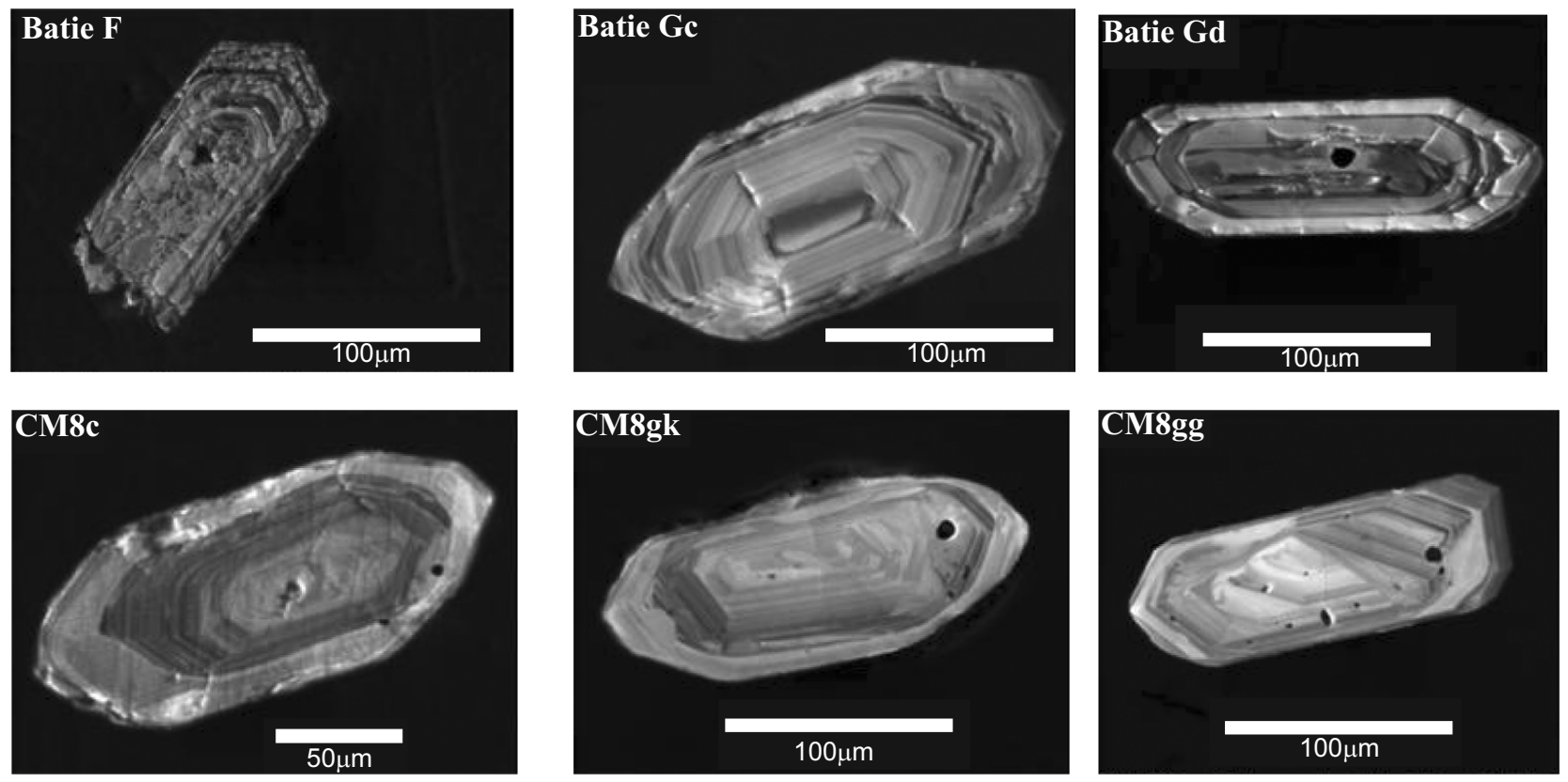

Fig. (11). SEM images of selected dated zircon grains in biotite- granite (sample Batié) and biotite-amphibole granite (sample CM8) showing oscillatory zoning, typical of magmatic growth.

metagreywacke sources. These source rocks are predominantly found in the upper part of the continental crust and we suggest that the source for the Bapa-Batié plutonic rocks was metamorphosed metagreywackes rocks. Presence of garnet in the residual phase requires that the partial melting process occurred at pressure higher than $5 \mathrm{~kb}$ (e.g. [54, 55]). Such source protolith with significant proportions of metasedimentary rocks was indicated for the high- $\mathrm{K}$ calc-alkaline plutons of the Borborema province in the NE Brazil [40, 41, 56, 57] and eastern Nigeria [36, 38, 58]. The high-K calc-alkaline to shoshonitic and metaluminous nature of the Bapa-Batié plutonic rocks require a metaluminous and relatively K-rich source [51, 59]. The differences observed in granites can be explained by differences in melting conditions and/or minor variation in source compositions. The low $\mathrm{Rb} / \mathrm{Sr}$ and the enrichment of LREE of the rocks are probably inherited from the source. Thus, the magmatism of the Bapa-Batié plutonic rocks may have involved remelting of a composite acid metagreywacke protolith in the upper crust. The low $\mathrm{Ni}$ and relative high $\mathrm{Cr}$ contents of the rocks are consistent with such a model. The large-scale melting of the source rocks could have been favoured by high heat flow during Pan-African orogenesis or underplating of mantle-derived magmas into the crust.

\section{Petrogenesis}

Field relations, petrographical features, whole rock major and trace element geochemistry are inconsistent with the derivation of the Bapa-Batié plutonic rocks from a common parental magma by simple crystal fractionation processes. Rather metasedimentary rocks are suggested as protoliths for these granitoids. As seen in Table 5, concentrations of $\mathrm{Zr}$ are high (117 to $315 \mathrm{ppm}$ in biotite- granite, 111 to $156 \mathrm{ppm}$ in biotite-amphibole granite, 276- $344 \mathrm{ppm}$ in granodiorite and
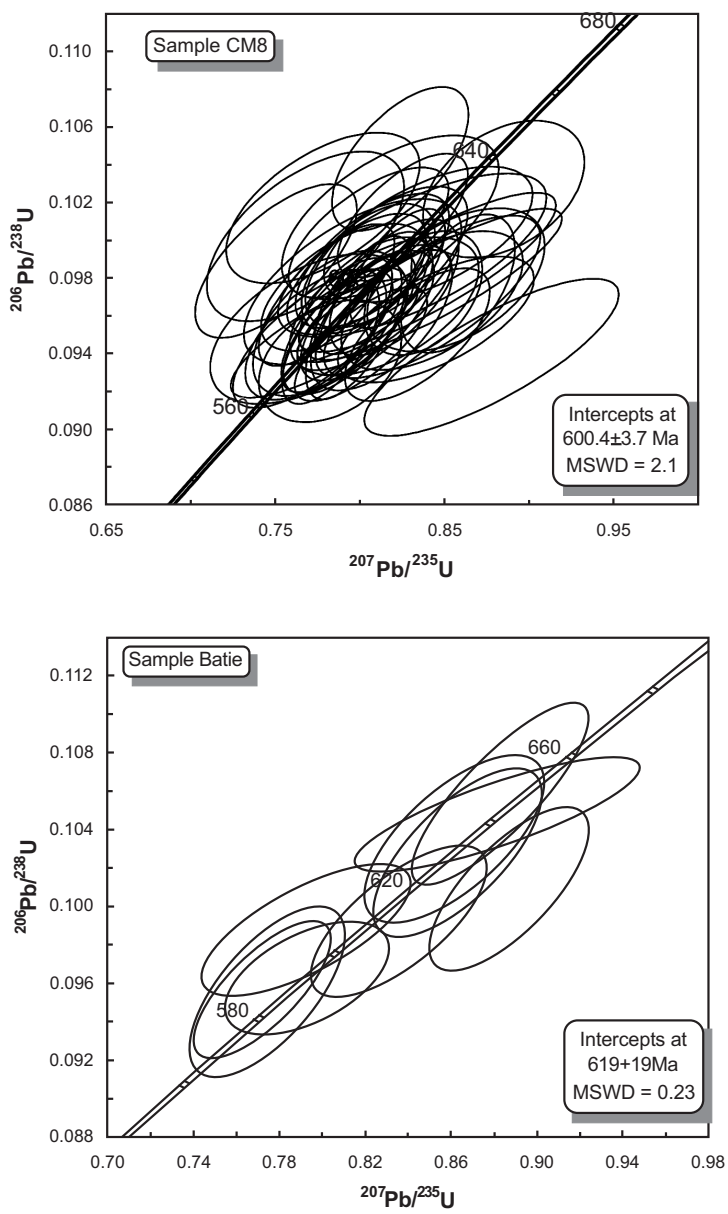

Fig. (12). U-Pb Concordia diagram for zircons from samples Batié and CM8. 
Table 7. LA-ICP-MS U-Pb Results for Zircons from Biotite- Granite of the Bapa-Batié Area

\begin{tabular}{|c|c|c|c|c|c|c|c|c|c|c|}
\hline \multirow[b]{2}{*}{ Sample Batié } & \multicolumn{4}{|c|}{ Isotopic Ratios } & \multicolumn{6}{|c|}{ Apparent Ages (Ma) } \\
\hline & $207 \mathrm{~Pb} / 235 \mathrm{U}$ & $1 \sigma \%$ & $206 \mathrm{~Pb} / 238 \mathrm{U}$ & $1 \sigma \%$ & $206 \mathrm{~Pb} / 238 \mathrm{U}$ & $1 \sigma \%$ & $207 \mathrm{~Pb} / 235 \mathrm{U}$ & $1 \sigma \%$ & $207 \mathrm{~Pb} / 206 \mathrm{~Pb}$ & Rho \\
\hline Batié Ga & 0.7887 & 1.8 & 0.0956 & 1.59 & 590 & 18 & 591 & 16 & 532 & 0.74 \\
\hline Batié A & 0.7743 & 1.9 & 0.0956 & 1.88 & 589 & 21 & 582 & 17 & 568 & 0.67 \\
\hline Batié Ga" & 0.772 & 1.7 & 0.0957 & 1.5 & 589 & 17 & 581 & 15 & 522 & 0.74 \\
\hline Batié Gb' & 0.7927 & 2 & 0.0963 & 1.25 & 593 & 14 & 594 & 18 & 542 & 0.52 \\
\hline Batié $\mathrm{Gb}$ & 0.7926 & 2.5 & 0.0988 & 1.42 & 611 & 16 & 594 & 22 & 562 & 0.71 \\
\hline Batié Ga' & 0.8352 & 2 & 0.0992 & 1.61 & 612 & 19 & 623 & 20 & 660 & 0.71 \\
\hline Batié F & 0.8868 & 1.7 & 0.1009 & 1.72 & 620 & 20 & 645 & 16 & 656 & 0.72 \\
\hline Batié D & 0.8629 & 1.8 & 0.1028 & 1.76 & 631 & 21 & 632 & 17 & 606 & 0.7 \\
\hline Batié A & 0.8611 & 2 & 0.1035 & 1.73 & 635 & 21 & 631 & 19 & 620 & 0.68 \\
\hline Batié Gc' & 0.8812 & 3.1 & 0.1048 & 1.13 & 645 & 14 & 642 & 30 & 620 & 0.82 \\
\hline Batié $\mathrm{C}$ & 0.8829 & 1.9 & 0.1059 & 1.81 & 649 & 22 & 643 & 18 & 598 & 0.84 \\
\hline Batié Gc & 0.9585 & 1.7 & 0.1062 & 1.14 & 653 & 14 & 687 & 17 & 740 & 0.62 \\
\hline
\end{tabular}

229-310 in fine-grained granite) in the investigated granitoids indicating high temperature $\left(741\right.$ to $\left.838^{\circ} \mathrm{C}\right)$ for their formation. Harris et al. (1993) [60] found that high $\mathrm{Rb} / \mathrm{Sr}$ (4-10) suggests granite formation by dehydration melting, whereas $\mathrm{Rb} / \mathrm{Sr}$ ratios $<4$ suggest vapor-present melting. Accordingly, it appears that most of the investigated granitoids ( $\mathrm{Rb} / \mathrm{Sr}<4$; Table 5) formed by vapor-present melting. Lower $\mathrm{Rb} / \mathrm{Sr}$ ratios < 4 suggests the participation of biotite during melt production of the granitoids [61, 62]. The strong positive correlation between $\mathrm{Zr} v s \mathrm{Sr}$ and $\mathrm{TiO}_{2}$ vs $\mathrm{Sr}$ in the Bapa-Batié plutonic rocks (Fig. 14) is best explained by different degrees of partial melting due to increasing temperature $[63,64]$.

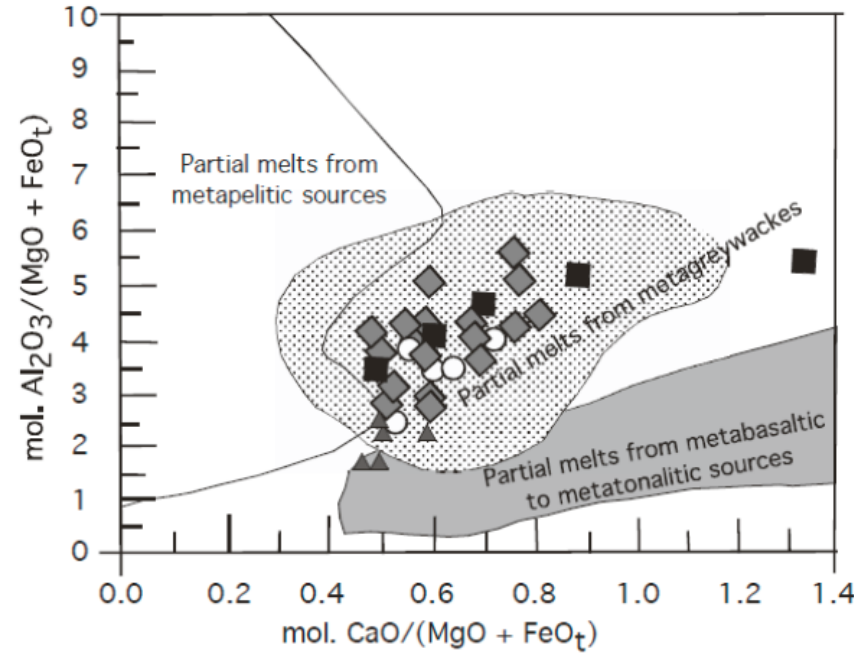

Fig. (13). Molar $\mathrm{CaO} /\left(\mathrm{MgO}+\mathrm{FeO}_{\mathrm{t}}\right)$ vs $\mathrm{Al}_{2} \mathrm{O}_{3} /\left(\mathrm{MgO}+\mathrm{FeO}_{\mathrm{t}}\right)$ for the Bapa-Batié granitoids. Symbols as in Fig. (4).

\section{Implications for the Assembly of Western Gondwana}

The results of this study and the recent works by Pimentel et al. (2001) [65], Piuzana et al. (2003) [66], Neves et al., 2006 [43] and Ferré et al. (2002) [37] on the geodynamic evolution of NE and Central Part of Brazil and
Nigeria, respectively, strengthen the previous suggestion [4, $9,11,21]$ that these belts shared a common evolution throughout most of the Proterozoic. Common features include (i) distensive Palaeoproterozoic crust (ca. 2.1Ga), (ii) dominance of metasedimentary sequences with Neoproterozoic deposition ages, (iii) ubiquitous presence of flat-lying structures of Neoproterozoic age ( 650-600 Ma), and, (iv) dominance of transpressional/transcurent deformation After $600 \mathrm{Ma}$. The lack of evidence for closure of wide oceanic domains in all these regions does not support the interpretation of the central and south domains of the PANEFB as a series of amalgamated terranes [18]. Destabilisation of a pre-existing continent that formed at the end of Paleoproterozoic/Eburnean orogeny [67] provides the simplest explanation to the above findings. An ultimate period of plate-wide extension occurred in the mid/late Neoproterozoic. This was immediately followed by convergence and contractional deformation marking the beginning of the Pan-African/Brasiliano orogeny, which essentially occurred in an intracontinental setting.

\section{CONCLUSION}

1. Plutonic rocks from the Bapa-Batié area are high-K, calc-alkaline to shoshonitic syntectonic intrusions that were emplaced during a Neoproterozoic event at 600-619Ma in Cameroon, in close spatial association with the Central Cameroon Shear Zone.

2. The elongate form of the plutons, consistent with the regional strike, demonstrates syn-kinematic magma emplacement during the Pan-African orogeny.

3. The data presented in this study are consistent with magmatism that may have involved remelting (biotite dehydration melting) of a composite metagreywacke protolith in the upper crust in the Central domain of the PANEFB.

4. Structural and geochemical characteristics indicate that the Bapa-Batié complex is part of the syn-collisional 
Table 8. LA-ICP-MS U-Pb Results for Zircons from Biotite-Amphibole Granite of the Bapa-Batié Area

\begin{tabular}{|c|c|c|c|c|c|c|c|c|c|c|c|}
\hline CM8 c" & 0.8776 & 3.5 & 0.0938 & 1.81 & 578 & 20 & 640 & 33 & 764 & 98 & 0.8 \\
\hline CM8Gi" & 0.8005 & 2 & 0.0945 & 1.3 & 582 & 14 & 597 & 18 & 656 & 58 & 0.74 \\
\hline CM8Gf' & 0.7826 & 1.5 & 0.0946 & 1.41 & 582 & 16 & 587 & 13 & 560 & 52 & 0.65 \\
\hline CM8 j' & 0.7788 & 2.9 & 0.0948 & 1.35 & 584 & 15 & 585 & 26 & 610 & 84 & 0.8 \\
\hline CM8Gi' & 0.7738 & 2.2 & 0.095 & 1.48 & 585 & 17 & 582 & 19 & 556 & 78 & 0.59 \\
\hline CM8Gc & 0.8338 & 2.1 & 0.095 & 1.51 & 585 & 17 & 616 & 20 & 692 & 68 & 0.65 \\
\hline CM8Gj" & 0.7914 & 1.9 & 0.0952 & 1.44 & 586 & 16 & 592 & 17 & 570 & 64 & 0.66 \\
\hline CM8 i & 0.7699 & 3.1 & 0.0953 & 1.7 & 587 & 19 & 580 & 28 & 526 & 118 & 0.51 \\
\hline CM8Gi & 0.7992 & 1.6 & 0.0959 & 1.84 & 590 & 21 & 596 & 15 & 580 & 62 & 0.68 \\
\hline CM8 i & 0.8126 & 2.1 & 0.0959 & 1.64 & 590 & 19 & 604 & 19 & 570 & 74 & 0.6 \\
\hline $\mathrm{CM} 8 \mathrm{Ge}$ & 0.8043 & 2 & 0.096 & 1.46 & 591 & 16 & 599 & 18 & 564 & 62 & 0.68 \\
\hline CM8Gh & 0.8032 & 1.5 & 0.0961 & 1.38 & 592 & 16 & 599 & 14 & 586 & 48 & 0.68 \\
\hline CM8 $\mathrm{f}^{\prime}$ & 0.8083 & 2.3 & 0.0963 & 1.35 & 593 & 15 & 602 & 21 & 588 & 68 & 0.76 \\
\hline CM8 e' & 0.8143 & 2.8 & 0.0965 & 1.55 & 594 & 18 & 605 & 25 & 604 & 100 & 0.54 \\
\hline CM8 e & 0.8425 & 2.6 & 0.0968 & 1.55 & 596 & 18 & 621 & 24 & 642 & 92 & 0.57 \\
\hline CM8Gg' & 0.8035 & 2 & 0.0969 & 1.37 & 596 & 16 & 599 & 18 & 594 & 68 & 0.61 \\
\hline CM8 b' & 0.8425 & 3.1 & 0.097 & 1.81 & 597 & 21 & 621 & 29 & 628 & 92 & 0.74 \\
\hline CM8Gg & 0.7802 & 2.1 & 0.0971 & 1.58 & 598 & 18 & 586 & 19 & 476 & 62 & 0.74 \\
\hline CM8 c' & 0.7956 & 3.3 & 0.0978 & 1.83 & 601 & 21 & 594 & 30 & 514 & 114 & 0.63 \\
\hline CM8 c"' & 0.8566 & 2.5 & 0.0979 & 1.58 & 602 & 18 & 628 & 23 & 594 & 96 & 0.48 \\
\hline CM8Ge' & 0.7978 & 2.1 & 0.0982 & 1.33 & 604 & 15 & 596 & 19 & 544 & 72 & 0.63 \\
\hline CM8 d' & 0.8397 & 3.7 & 0.0983 & 1.75 & 605 & 20 & 619 & 34 & 608 & 102 & 0.84 \\
\hline CM8Gg" & 0.822 & 1.4 & 0.0985 & 1.31 & 606 & 15 & 609 & 13 & 600 & 48 & 0.65 \\
\hline CM8Gb' & 0.8048 & 2 & 0.0985 & 1.42 & 605 & 16 & 600 & 18 & 612 & 72 & 0.56 \\
\hline CM8Gd & 0.8072 & 2.3 & 0.0989 & 1.47 & 608 & 17 & 601 & 20 & 576 & 76 & 0.64 \\
\hline $\mathrm{CM} 8 \mathrm{c}$ & 0.7503 & 2.6 & 0.0989 & 1.68 & 608 & 20 & 568 & 22 & 402 & 86 & 0.68 \\
\hline CM8 d & 0.8156 & 2.4 & 0.0991 & 1.54 & 609 & 18 & 606 & 22 & 518 & 84 & 0.62 \\
\hline CM8 g' & 0.8308 & 3.9 & 0.0992 & 1.78 & 610 & 21 & 614 & 36 & 578 & 128 & 0.7 \\
\hline CM8 b & 0.7677 & 3.1 & 0.1002 & 1.83 & 615 & 22 & 578 & 27 & 378 & 114 & 0.58 \\
\hline CM8 a" & 0.8402 & 3 & 0.1003 & 1.46 & 616 & 17 & 619 & 28 & 594 & 106 & 0.59 \\
\hline $\mathrm{CM} 8 \mathrm{~Gb}$ & 0.805 & 2.4 & 0.1004 & 1.4 & 617 & 17 & 600 & 22 & 528 & 80 & 0.67 \\
\hline $\mathrm{CM} 8 \mathrm{~b}$ & 0.8178 & 3.2 & 0.1012 & 1.75 & 621 & 21 & 607 & 29 & 502 & 114 & 0.59 \\
\hline CM8Gd' & 0.8252 & 1.9 & 0.1013 & 1.32 & 622 & 16 & 611 & 18 & 556 & 64 & 0.67 \\
\hline CM8 g & 0.778 & 3 & 0.1017 & 1.6 & 624 & 19 & 584 & 27 & 450 & 112 & 0.57 \\
\hline CM8Gc' & 0.8848 & 2.2 & 0.1027 & 1.44 & 630 & 17 & 644 & 21 & 716 & 92 & 0.39 \\
\hline CM8 j" & 0.8237 & 2 & 0.1043 & 1.49 & 640 & 18 & 610 & 18 & 500 & 70 & 0.61 \\
\hline
\end{tabular}


magmatism emplaced in the course of Pan-African deformation during late-orogenic uplift and extension. The plutonic rocks of the Bapa-Batié area are very similar to other Neoproterozoic high-K calc-alkaline syntectonic plutons from western and central east Cameroon in modal, major and trace element characteristics. Most of these features are found in the Borborema Province of NE Brazil [43] and in the Nigeria Province [37], indicating a shared evolution during most of the Proterozoic. Roughly similar syntectonic plutonism in relation with lithospheric-scale shear zone is also observed in NE Brazil, especially close to the Pernambuco Shear Zone.
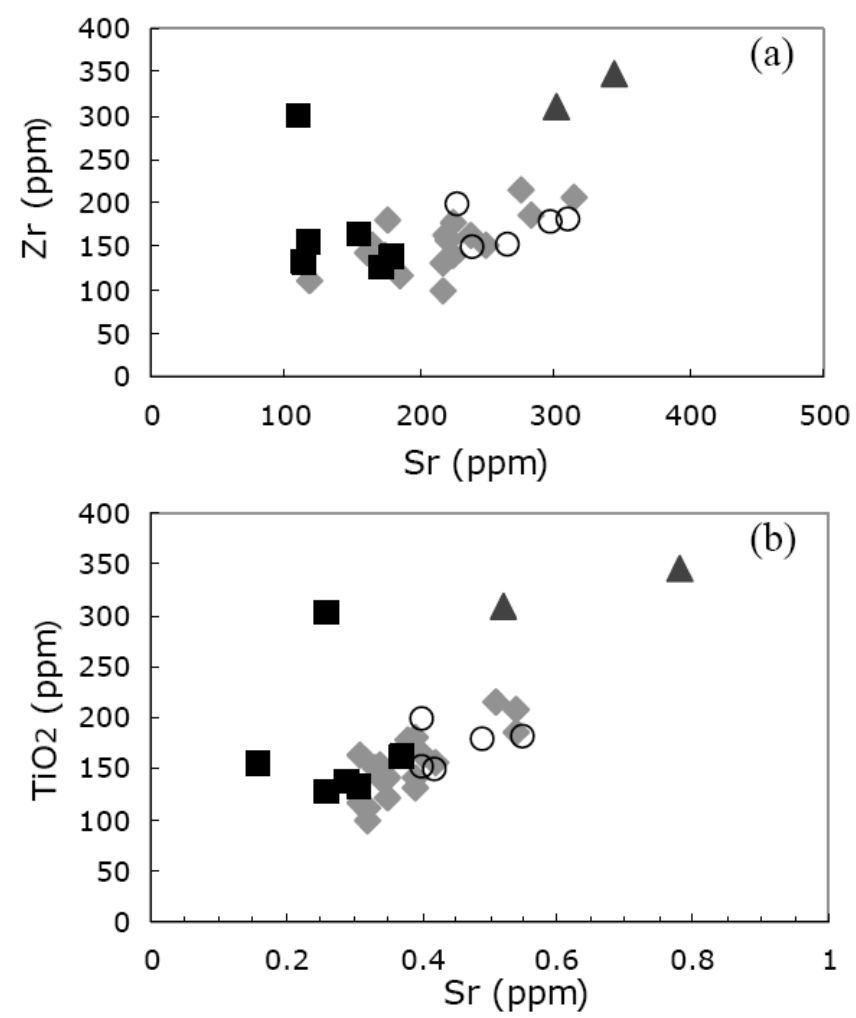

Fig. (14). $\mathrm{Zr}$ vs $\mathrm{Sr}$ and $\mathrm{TiO}_{2}$ vs $\mathrm{Sr}$ variation diagrams for the BapaBatié granitoids. Symbols as in Fig. (4).

\section{ACKNOWLEDGEMENTS}

Thanks are due to Professor L. Baumgartner, Institute of Mineralogy and Geochemistry, University of Lausanne, Switzerland for providing many facilities.

\section{REFERENCES}

[1] B. Barbarin, "A review of the relationships between granitoids types, their origins and their geodynamic environments", Lithos, vol. 46, pp. 605-626, 1999.

[2] D.H.W. Hutton, and T.J. Dempster, P.E. Brown, S.D. Becker, “A new mechanism of emplacement: intrusion in active extensional shear zones", Nature, vol. 343, pp. 452-455,1990.

[3] J.P. Nzenti, "L'Adamaoua panafricain (région de Banyo) : une zone clé pour un modèle de la chaîne panafricaine nordéquatoriale au Cameroun", Thèse Doct. D'Etat, Univ Cheikh Anta Diop -Univ Nancy I, France, 1998.

[4] J.P. Nzenti, P. Barbey, J.M. Bertrand, and J. Macaudière, "La chaîne panafricaine au Cameroun : cherchons suture et modèle!" In: S. G. F. Ed. $15^{\text {ème }}$ Réunion des Sciences de la Terre, Nancy, France, pp. 99, 1994.

[5] J.P. Nzenti, B. Kapajika, G. Wörner, and R.T. Lubala "Synkinematic emplacement of granitoids in a Pan-African shear zone in Central Cameroon", J. Afr. Earth Sci., vol. 45, pp. 74-86, 2006.

[6] J.P. Nzenti, E.L. Tankonjiosseu, and A. Nzina Nchare, "The metamorphic evolution of the Paleoproterozoic high grade Banyo gneisses (Adamawa, Cameroon, Central Africa)", J. Camer. Acad. Sci., vol. 7, pp. 95-109, 2007.

[7] T. Ngnotué, J.P. Nzenti, P. Barbey, and F.M. Tchoua "The NtuiBétamba high-grade gneisses: a northward extension of the PanAfrican Yaoundé gneisses in Cameroon", J. Afr. Earth Sci., vol. 31, pp. 369-381, 2000.

[8] V. Ngako, P. Afrfaton, J.M. Nnange, T. Njanko, "Pan-African tectonic evolution in central and southern cameroon : transpression and transtension during sinistral shear movements", J. Afr. Earth Sci., vol. 36, pp. 207-214, 2003.

[9] E.L. Tankonjiosseu, J.P. Nzenti, T. Njanko, B. Kapajika, and A. Nédelec, "New U-Pb zircon ages from Tonga (Cameroon): coexisting Eburnean-Transamazonian $(2.1 \mathrm{Ga})$ and Pan-African (0.6 Ga) imprints", C. R. Géosci., vol. 337, pp. 551-562, 2005.

[10] S.F. Toteu, J. Penaye, Y.H. Djomani Poudjom, "Geodynamic evolution of the Pan-African belt in central Africa with special reference to Cameroon," Can. J. Earth Sci., vol. 41, pp. 73-85, 2004.

[11] J.P. Nzenti, P. Barbey, J. Macaudière, and D. Soba, "Origin and evolution of late Precambrian high-grade Yaoundé gneisses (Cameroon)", Precam. Res., vol. 38, pp. 91-109, 1988.

[12] J. Penaye, S.F. Toteu, W.R. Van Schmus, J.P. Nzenti, "U-Pb and $\mathrm{Sm}-\mathrm{Nd}$ preliminary geochronologic data on the Yaoundé series, Cameroon: re-interpretation of the granulitic rocks as the suture of a collision in the centrafrican belt", C. R. Acad. Sci. Paris, vol. 317, pp. 789-794, 1993.

[13] J.P. Nzenti, "Neoproterozoic alkaline meta-igneous rocks from the Pan-African North Equatorial fold belt (Yaoundé, Cameroon): biotitites and magnetite rich pyroxenites", J. Afr. Earth Sci., vol. 26, pp. 37-47, 1998.

[14] J.P. Nzenti, P. Barbey, P. Jegouzo, and C. Moreau, "Un nouvel exemple de ceinture granulitique dans une chaîne Protérozoïque de collision: les migmatites de Yaoundé au Cameroun", C. R. Acad. Sci., vol. 299/17, pp. 1197-1199, 1984.

[15] C. Castaing, J.L. Feybesse, D. Thiéblemont, C. Triboulet, and P. Chèvremont, "Palaeogeographical reconstructions of the PanAfrican/Brasiliano orogen: closure of an oceanic domain or intracontinental convergence between major blocks?" Precam. Res., vol. 69, pp. 327-344, 1994.

[16] B.B Brito Neves, W.R. Van Schmus, A. Fetter, "North-western Africa-North eastern Brazil : major tectonic links and correlation problems", J. Afr. Earth Sci., vol. 34, pp. 275-278, 2002.

[17] U.G. Cordani, M.S. D'Agrella-Filho, B.B. Brito-Neves, R.I.F. Trindale, "Tearing up Rodinia: the Neoproterozoic palaeogeography of South American cratonic fragments", Terra Nova, vol. 15, pp. 350-359, 2003.

[18] E. Njonfang, V. Ngako, C. Moreau, P. Affaton, H. Diot, "Restraining bands in high temperature shear zones: The "Central Cameroon Shear Zone", Central Africa", J. Afr Earth Sci., vol. 52, pp. 9-20, 2008.

[19] T.C. Nguiessi, J.P. Nzenti, E.N. Nsifa, P. Tempier, and F.M. Tchoua, "Les granitoïdes calco-alcalins, syncisaillement de Bandja dans la chaîne pana fricaine nord-équatoriale au Cameroun", $C . R$. Acad. Sci., vol. 325, pp. 95-101, 1997.

[20] G. Tagne-Kamga, E. Mercier, M. Rossy, and N.E. Nsifa Synkinematic emplacement of the Pan-African Ngondo igneous complex (west Cameroon, central Africa)," J. Afr. Earth Sci., vol. 28, pp. 675-691, 1999

[21] C. Nzolang, H. Kagami, J.P. Nzenti, and F. Holtz, "Geochemistry and preliminary $\mathrm{Sr}-\mathrm{Nd}$ isotopic data on the Neoproterozoic granitoids from the Bantoum area, west Cameroon: evidence for a derivation from a Paleoproterozoic Archean crust", Polar Geosci., vol.16, pp. 196-226, 2003.

[22] M. L. Djouka-Fonkwé, B. Schulz, U. Schüssler, J-P. Tchouankoué, and C. Nzolang, "Geochemistry of the Bafroussam Pan-African Iand S- type granitoids in western Cameroon", J. Afr. Earth Sci., vol. 50, vol., pp. 148-167, 2008.

[23] A. Ganwa, W. Frisch, W. Siebel, S.K. Cosmas, O.J. Mvondo, M Satir, N.J. Tchakounté, "Zircon ${ }^{207} \mathrm{~Pb}-{ }^{206} \mathrm{~Pb}$ evaporation ages of Pan-African metasedimentary rocks in the Kombé-II area (Bafria Group, Cameroon): constraints on protolith age and provenance", J. Afr. Earth Sci., vol. 51, pp. 77-88, 2008. 
[24] D. Tchaptchet Tchato, B. Schulz and J.P. Nzenti, "Electron microprobe (EMP) monazite dating and P-T data of the Neoproterozoic metamorphic and mylonitic events in the Kekem area, Cameroon North Equatorial Fold belt", N. Jb. Miner. Abh., vol.186, Issue1, pp. 95-109, 2009.

[25] S.F Toteu, A. Michard, J. M. Bertrand, and G. Rocci, "U/Pb of Precambrian rocks from northern Cameroon, orogenic evolution and chronology of the Panafrican belt of central Africa", Precamb. Res., vol. 37, pp.71-87, 1987.

[26] G. Njiekak, W. Dörr, J-P. Tchouankoué, and G. Zulauf, "U-Pb zircon and microfabric data of (meta) granitoids of western Cameroon: constraints on the timing of pluton emplacement and deformation in the Pan-African belt of Central Africa", Lithos, vol. 102, pp. 460-477, 2008.

[27] C. Chebeu, "Igneous emplacement in a transpressive shear zone: Bapa-Batié granitoids (West Cameroon)", MSc thesis, Univ Yaoundé I, p. 52, 2003.

[28] B. Kankeu, "Anisotropie de la susceptibilité magnétique (ASM) et fabriques des roches Néoprotérozoiques des régions de GargaSarali et Bétaré-Oya à l'Est Cameroon: implications géodynamiques pour l'évolution de la chaîne panafricaine d'Afrique Centrale", Ph.D. thesis. Université de Yaoundé I, p. 232, 2008.

[29] B. Kankeu, O.R. Greiling, and J.P. Nzenti, "Pan-African strike-slip tectonics in eastern Cameroon. Magnetic fabrics (AMS) and structure in the Lom basin and its gneissic basement (Bétaré Oya)", Precambr. Res., vol. 17, no. 3-4, pp. 258-272, 2009.

[30] G. Witt-Eickschen, H.A. Seck, K. Mezger, S.M. Eggins, and R. Altheer, "Lithospheric mantle evolution beneath the Eifel (Germany): constraints from $\mathrm{Sr}-\mathrm{Nd}-\mathrm{Pb}$ isotopes and trace element abundances in spinel peridotite and pyroxenites xenoliths", $J$. Petrol., vol. 44, pp. 1077-1095, 2003.

[31] K. R. Ludwig, User's Manual for Isoplot/Ex v. 3.00. A Geochronological Toolkit for Microsoft Excel, BGC Special Publication 4, Berkeley, p. 71, 2003.

[32] F. Debon, and M. Lemmet, "Evolution of $\mathrm{Mg} / \mathrm{Fe}$ ratios in late Variscan plutonic rocks from the external crystalline massifs of the Alps (France, Italy, Switzerland)", J. Petrol., vol. 40, pp. 1151$1185,1999$.

[33] B.R. Frost, C.G. Barnes, W.J. Collins, R.J. Arculus, D.J. Ellis, and C.D. Frost, "A geochemical classification for granitic rocks", $J$. Petrol., vol. 42, pp. 2033-2048, 2001.

[34] J.M. Bertrand, C. Dupuy, J. Dostal, and I. Davidson, "Geochemistry and geotectonic interpretation of granitoids from Central Iforas (Mali, West Africa)", Precamb. Res., vol. 26, pp. 265-283, 1984.

[35] R. Ayuso, and J.G. Arth, "The Northeast Kingdom batholith, Vermon: magmatic evolution and geochemical constraints on the origin of Acadian granitic rocks", Contrib. Miner. Petrol., vol. 111, pp. 1-23, 1992.

[36] S.S. Dada, L. Briqueu, U. Harms, J.R. Lancelot, and G. Matheis, "Charnockitic and monzonitic Pan-African series from northcentral Nigeria: trace-element and $\mathrm{Nd}, \mathrm{Sr}, \mathrm{Pb}$ isotope constraints on their petrogenesis”, Chem. Geol., vol. 124, pp. 233-252, 1995.

[37] E. Ferré, G. Gleizes, and R. Caby, "Obliquely convergent tectonics and granite emplacement in the Trans-Saharan belt of eastern Nigeria: a synthesis", Precambr. Res., vol. 114, pp. 199-219, 2002.

[38] V.O. Olarewaju, and M.A. Rahaman, "Petrology and geochemistry of older granites from some parts of northern Nigeria", Niger. J. Min. Geol., vol. 18, pp. 16-28, 1982.

[39] V.P. Ferreira, A.N. Sial, M.M. Pimentel, and C.A.V. Moura, "Intermediate to acidic magmatism and crustal evolution in the Transversal Zone, north-eastern Brazil”, In: V. Mantesso-Neto, A. Bartorelli, C. Carneiro, B.B. Brito-Neves, Eds., Geologia do continente Sul-Americano: evolução da obra de Fernando Flàvio Marques de Almeida. Beca, São Paulo, Brazil, 2004, pp. 189-201.

[40] G. Mariano, P.B. Correia, S.P. Neves, and A.F.D. Silva Filho, "The high-K calc-alkaline Alagoinhas pluton: anisotropy of magnetic susceptibility, geochemistry, emplacement setting, and implications for the evolution of Borborema Province, NE Brazil", Int. Geol. Rev., vol. 51, pp. 502-519, 2009.

[41] S. P. Neves, and G. Mariano, "High-K calc-alkalic plutons in NE Brazil: origin of the biotite diorite/quartz monzonite to granite association and implications for the evolution of the Borborema Province", Inter. Geol. Rev., vol. 39, pp. 621-638, 1997.
[42] S.P. Neves, A. Vauchez, and C.J. Archanjo. "Shear zone-controlled magma emplacement or magma-assisted nucleation of shear zones"? Insights from northeast Brazil. Tectonophysics, vol. 262, pp. 349-364, 1996.

[43] S. P. Neves, O. Bruguier, A. Vauchez, D. Bosch, J.M.R Silva, and G. Mariano, "Timing of crust formation, deposition of supracrustal sequences, and Transamazonian and Brasiliano metamorphism in the East Pernambuco belt (Borborema Province, NE Brazil): Implications for western Gondwana assembly", Precambr. Res., vol. 149, pp. 197-216, 2006.

[44] J.A. Pearce, "Trace element characteristics of lavas from destructive plate boundaries", in R.S. Thorpe, Ed., Andesites. London, John Wiley, 1982, pp. 525-548.

[45] T.M. Harrison, and B. Watson, "Kinetics of zircon dissolution and zirconium diffusion in granitic melts of variable water content". Contrib. Miner. Petrol., vol. 84, pp. 66-72, 1983.

[46] E.B. Watson, and T.M. Harrison, "Zircon saturation revisited: temperature and composition effects in a variety of crustal magma types", Earth Planetary Sci Lett, vol. 64, pp. 295-304, 1983.

[47] B.W. Chappell, and A.J.R.White, "Two contrasting granite types", Pacific Geol., vol. 8, pp. 173-174, 1974.

[48] B.W. Chappell, and A.J.R.White, "I- and S-type granites in the Lachlan Fold Belt", Trans. Roy. Soc. Edinburgh, vol. 83, pp. 1-12, 1992.

[49] F.F. Altherr, A. Holl, E. Hegner, C. Langer, and H. Kreuzer, "High-potassium, calc-alkaline I-type plutonism in the European variscides: northern Vosges (France) and northern Schwarzwald (Germany)", Lithos, vol. 50, pp 51-73, 2000.

[50] D.J. De Paolo, "A neodymium and strontium isotopic study of the Mesozoic calc-alkaline granitic batholiths of the Sierra Nevada and Peninsular Ranges, California”, J. Geophys. Res, vol. 86 (B11), pp. 10470-10488, 1981.

[51] M.P. Roberts, and J.D. Clemens, "Origin of high-potassium, calcalkaline, I-type granitoids”, Geology, vol. 21, pp. 825-828, 1993.

[52] J.P. Liégeois, R. Black, J. Navez, and L. Latouche "Early and late pan African orogenies in the Airr assembly of terranes (Tuareg Shield, Niger)", Precamb. Res., vol. 67, pp. 59-88, 1994.

[53] M.P. Roberts, C. Pin, J.D. Clemens, and J.L. Paquette, "Petrogenesis of mAfric to felsic plutonic rock associations: the calc-alkaline Quérigut complex, French pyrénées", J. Petrol., vol. 41, issue 6, pp. 808-844, 2000.

[54] J. M. Montel, and D. Vielzeuf, "Partial melting of metagreywackes. Part 2. Compositions of mineral and melts", Cont. Mineral. Petrol., vol. 128, pp. 176-196, 1997.

[55] A.E Patiño Douce, and J. S. Beard, "Effects of $\mathrm{P}, \mathrm{f}\left(\mathrm{O}_{2}\right)$ and $\mathrm{Mg} / \mathrm{Fe}$ ratio on dehydratation melting of model metagreywackes", $J$. Petrol., vol. 37, pp. 999-1034, 1996.

[56] A.F.D. Silva Filho, I.P. Guimaraes, M.F.L.D. Brito, and M.M. Pimental, "Geochemical signatures of main Neoproterozoic Latetectonic granitoids from the Proterozoic Sergipano fold belt, Brazil: significance for the Brasiliano orogeny", Intern. Geol. Rev., vol. 39, pp 639-659, 1997.

[57] S. P. Neves, and G. Mariano, "The lithospheric mantle as a source of magmas during orogenic processes: insights from high-K diorites in the Borborema Province and implications for continental dynamics", J. Virt. Expl., vol. 17, pp. 1-14, 2004.

[58] E. Ferré, R. Caby, J.J. Peucat, R. Capdevila, and P. Monié, "PanAfrican, post-collisional, ferro-potassic granite and quartzmonzonite plutons of eastern Nigeria", Lithos, vol. 45, pp. 255-279, 1998.

[59] K.T. Winther, and R.C. Newton, "Experimental melting of hydrous low-K tholeiites: evidence of the origin of Archean cratons", Geol. Soc. Denmark Bull., vol. 39, pp. 213-228, 1991.

[60] N.B.W. Harris, S. Inger, and J. Massey, "The role of fluids in the formation of High Himalayan leucogranites". In: Treloar PJ, Searle MP (eds) Himalayan tectonics. Spec. Publ. Geol. Soc. Lond., vol. 74, pp. 391-400, 1993.

[61] S. Inger, and N.B.W. Harris, "Geochemical constraints on leucogranites magmatism in the Lantang valley, Nepal Himalaya", J. Petrol,. 34, 345-368, 1993.

[62] T.M. Harrisson, M. Grove, K.D. McKeegan, C.D. Coath, O.M. Lovera, and P. Le Fort, "Origin and episodic emplacement of the Manaslu intrusive complex, central Himalaya", J. Petrol., vol. 40, pp. 3-19, 1999.

[63] D. Visona, and B. Lombardo, "Two micas and tourmaline leucogranites from the Everest-Macalu region (Nepal-Tibet). 
Himalayan leucogranites genesis by isobaric heating?" Lithos, vol. 62, pp. 125-150, 2002.

[64] A. M. Moghazi, M. A. Hassanen, F. H. Mohamed, and S. Ali, "Late Neoproterozoic strongly peraluminous leucogranites, South Eastern Desert, Egypt. Petrogenesis and geodynamic significance", Mineral. Petrol., vol. 81, pp. 19-41, 2004.

[65] M. M. Pimentel, M. A. Dardenne, R. A. Fuck, M. G. Viana, S.L. Junges, D. P. Fischel, H.J. Seer, and E. L. Dantas, "Nd isotopes and the provenance of detrital sediments of the Neoproterozoic Brasilia Belt, central Brazil", J. South Am. Earth Sci., vol. 14, pp. 571-585, 2001.

[66] D. Piuzana, M. M. Pimentel, R. A. Fuck, and R. Armstrong, "Neoproterozoic granulite facies metamorphism and coeval granitic magmatism in the Brasília Belt, central Brazil: regional implications of new SHRIMP U-Pb and Sm-Nd data", Precambr. Res. Vol. 125, pp. 245-273, 2003.

[67] J.J.W. Rogers, "A history of continents in the past three billion years", J. Geol., vol. 104, pp. 91-107, 1996.

[68] R. Caby, A.N. Sial, M. Arthaud, and A. Vauchez, "Crustal evolution and the Brasiliano orogeny in northeast Brazil.- in : R. D. Dallmeyer and J. P. Lécorché, Eds. The west African orogens and
circum-Atlantic correlatives", Springer-Verlag, Berlin, pp. 373397, 1991.

[69] D. Soba "La série de Lom: Etude Géologique et Géochronologique d'un bassin volcano-sédimentaire de la Chaîne PanAfricaine à l'Est du Cameroun", Thèse Doctorat D'Etat, Université de Paris VI, $198 \mathrm{p}, 1989$.

[70] A. L., Streckeisen, "Classification of the common igneous rocks by means of their chemical composition: a provisional attempt". Neues Jahrbuch fur Mineralogie, Monatshefte, vol. H. 1, pp. 1-15, 1976.

[71] R. W. Le Maitre, P. Bateman, A. Dudek, J. Keller, J. Lameyre, M. J. Le As, P.A. Sabine, R. Schmid, H. Sorensen, A. Streckeisen, A. R. Ooley, and B. Zanettin, "A classification of igneous rocks and glossary of terms", Blackwe11, Oxford, 1989.

[72] P.C. Rickwood, "Boundary lines within petrologic diagrams which use oxides of major and minor elements", Lithos, vol. 22, pp. 247$263,1989$.

[73] N.M. Evensen, M.J. Hamilton, and R.J. O’Nions, "Rare earth abundances in chondritic meteorites", Geochem Cosmochim Acta, vol. 42, pp. 1199-1212, 1978.

(C) Chebeu et al.; Licensee Bentham Open.

This is an open access article licensed under the terms of the Creative Commons Attribution Non-Commercial License (http://creativecommons.org/licenses/by-nc/ $3.0 /$ ) which permits unrestricted, non-commercial use, distribution and reproduction in any medium, provided the work is properly cited. 